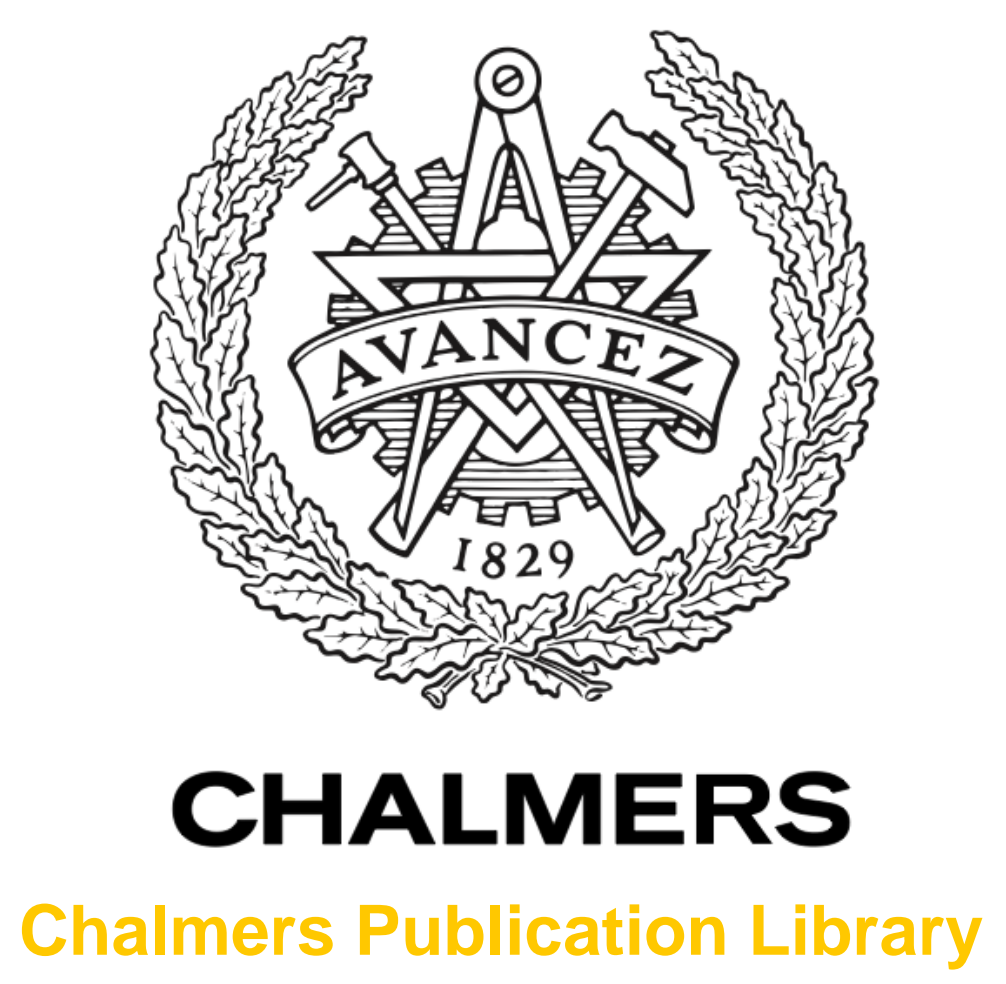

\title{
Load Management of Modular Battery using Model Predictive Control: Thermal and State-of-Charge Balancing
}

This document has been downloaded from Chalmers Publication Library (CPL). It is the author's version of a work that was accepted for publication in:

IEEE Transactions on Control Systems Technology (ISSN: 1063-6536)

Citation for the published paper:

Altaf, F. ; Egardt, B. ; Johannesson, L. (2016) "Load Management of Modular Battery using Model Predictive Control: Thermal and State-of-Charge Balancing". IEEE Transactions on

Control Systems Technology

http://dx.doi.org/10.1109/TCST.2016.2547980

Downloaded from: http://publications.lib.chalmers.se/publication/228087

Notice: Changes introduced as a result of publishing processes such as copy-editing and formatting may not be reflected in this document. For a definitive version of this work, please refer to the published source. Please note that access to the published version might require a subscription. 


\title{
Load Management of Modular Battery using Model Predictive Control: Thermal and State-of-Charge Balancing
}

\author{
Faisal Altaf, Bo Egardt, Fellow, IEEE, and Lars Johannesson
}

\begin{abstract}
Thermal and state-of-charge (SOC) imbalance is well known to cause non-uniform ageing in batteries. This paper presents the electro-thermal control of a multi-level converter (MLC) based modular battery to address this issue. The modular battery provides a large redundancy in synthesizing terminal voltage, which gives extra degrees-of-freedom in control on cell level. There are multiple tightly coupled control objectives including the simultaneous thermal and SOC balancing as well as battery terminal voltage control. The main purpose of this paper is to devise an electro-thermal control scheme for cases where full future driving information is not accessible. The control scheme is based on decomposition of controller into two orthogonal components, one for voltage control and the other for balancing control. This problem decomposition enables the application of constrained linear quadratic model predictive control scheme to solve the balancing problem elegantly. The control scheme is thoroughly evaluated through simulations of a four cell modular battery. The results show that a rather short prediction horizon is sufficient to achieve robust control performance.
\end{abstract}

Index Terms-Batteries, cell balancing, SOC balancing, thermal balancing, modular battery, battery control, multilevel converters, model predictive control (MPC).

\section{INTRODUCTION}

There has in recent years been an increasing interest in battery-powered electrified vehicles (xEVs) to reduce carbon footprint of transportation and the dependence on fossil fuels. The battery pack of $\mathrm{xEVs}$ is one of the most expensive but a key component in the powertrain, which helps to downsize or completely eliminate the internal combustion engine. Therefore, the battery lifetime is an important factor for the success of xEVs. In this regard, in addition to ongoing fundamental research on battery materials, batteries have also gained a lot of research attention recently from the systems and control community, see for example [1] and [2]. The overall goal is to develop a knowledge base to design battery health-conscious power management algorithms and battery management systems (BMS) for optimal utilization of currently available cells to guarantee their long and uniform lifetime in large-scale energy storage applications like xEVs and smart grids.

The battery pack of $\mathrm{xEVs}$ consists of long strings of series and parallel connected cells to meet the traction power demands. The lithium-ion batteries, due to their relatively higher specific energy and long deep-cycle life, are currently

Manuscript received April 26, 2015; revised November 29, 2015: accepted February 27, 2016. Date of publication XX XX 2016; date of current version March 24, 2016. Manuscript received in final form March 24, 2016. This work was supported by the Chalmers Energy Initiative, Sweden. Recommended by Associate Editor Subbarao Varigonda.

F. Altaf and B. Egardt are with Chalmers University of Technology, Automatic Control Group, Department of Signals and Systems, 41296 Gothenburg Sweden (e-mails: \{faisal.altaf, bo.egardt @ chalmers.se).

L. Johannesson was with Chalmers and now with Volvo Car Corp., Gothenburg Sweden (e-mail: lars.johannesson.mardh@volvocars.com). emerging as one of the major alternative choices for future xEVs. However, like all other battery types, the ageing rate of each Li-ion cell in a battery pack is greatly affected by various factors like SOC level, depth-of-discharge (DOD), temperature, and c-rate etc [3]-[6]. In short, the cells in the string being stored or cycled at higher SOC-level, DOD and temperature age faster than those at lower SOC, DOD, and temperature. Therefore, thermal, SOC, and DOD imbalances in a battery pack may cause nonuniform ageing of cells. Another serious issue is that the cell imbalance and nonuniform ageing are tightly coupled, which may lead to a vicious cycle resulting in the premature end of battery life. In addition to nonuniform ageing, the SOC imbalance also has a detrimental impact on the total usable capacity of the battery, see review papers [7] and [8] for details. Therefore, thermal and SOC imbalance can be considered as an indirect indication of either temporary or permanent health imbalance among cells.

Thermal, SOC and DOD imbalance is inevitable in battery packs of xEVs. The thermal imbalance is mainly caused by variations in internal resistances of cells and temperature gradient in the battery coolant, which is not negligible in xEVs [9][11]. The SOC imbalance is primarily caused by variations in capacities, leakage currents, and operating conditions of cells, whereas the DOD imbalance occurs as a result of the SOC and capacity imbalance. It is also pertinent to mention here that the the parametric variations are not negligible even in fresh cells of a same batch [12]. Thus, thermal and SOC balancer is quite critical for optimal performance of automotive batteries. In addition, the potential of used automotive battery packs (socalled second life batteries) is being investigated for smart grid energy storage applications [13]. The need of thermal and SOC balancer may be even more critical in such applications due to very high probability of large variance in parameters of these second-life batteries. The SOC balancing can be achieved using various types of passive or active SOC balancers, see [14][16], whereas thermal balancing can potentially be achieved using active cooling with reciprocating air-flow, see [11], but not under parametric variations [17].

The notion of simultaneous thermal and SOC balancing using a single active balancing device was introduced in our previous work, see [17]-[19]. A similar kind of conceptual study has also been carried out recently by other authors [20]. Thermal and SOC balancing are two tightly coupled and somewhat conflicting objectives, but it is possible to achieve both simultaneously in an average sense [8]. For this, load variations and surplus voltage in the battery pack are required. Also, a special balancing device that enables the non-uniform load scheduling of cells, is needed. The MLC-based modular battery system is a potential candidate for this purpose. The MLC [21], [22] consists of $n$ cascaded power units (PUs), 
each containing a smaller battery unit and a full-bridge dcdc converter, which enables bidirectional power flow from each battery unit. The modular battery is reconfigurable to generate a range of terminal voltages. It also provides a large redundancy in the voltage synthesis, which gives extra degrees-of-freedom in control.

The modular battery has multiple electro-thermal control objectives, including simultaneous thermal and SOC balancing as well as terminal voltage control. The control problem of modular battery boils down to the load management problem. The main goal of BMS is to satisfy the demand without draining out (SOC imbalance) and over-stressing (thermal imbalance) any single battery unit. Since cells are assumed to have variations in parameters (health imbalance) and operating conditions, the equal loading of cells is not a good policy for optimal battery performance. A natural question is then how to distribute demanded load among cells. Our approach is to use a criterion based on level of thermal and SOC deviations. Indeed, the electro-thermal control problem of the modular battery can be formulated and solved off-line as a constrained convex optimization problem [17]-[19]. The optimizer decides the power flow out of (or into) each cell at each time instant to meet the total driving (or regenerating) power demand such that all cells remains balanced in terms of SOC and temperature gauges. The solution is globally optimal if full future driving information (i.e. load demand) is accessible.

However, in most practical applications, full drive cycle is hardly known a priori. Since it may still be possible to achieve load predictions over short horizon, this study employs the model predictive control (MPC) framework [23] to solve battery load management problem. The main purpose is to devise a predictive control scheme that achieves the balancing objectives by using load forecast over a very short horizon. In addition, we also aim to achieve balancing objectives without imposing any hard constraints on cell temperatures and SOCs to secure feasibility. The main idea in this regard is to reformulate the original control problem as a controlconstrained linear quadratic (LQ) problem and then solve it in an MPC framework with a short prediction horizon. The control problem is not readily on standard LQ form. Therefore, the problem transformation to standard form is carried out carefully based on the decomposition of controller into two orthogonal components, one for voltage control and the other for balancing control. The new control scheme uses a simple voltage controller to strictly satisfy the load voltage demand. After this, any redundancy available in the modular battery is then optimally used by the balancing controller (LQ MPC) to achieve thermal and SOC balancing as an add-on benefit without disturbing the voltage. The novel way of decomposing voltage and balancing control tasks into two separate subtasks is one of the main contribution of this paper. This control decomposition reveals the controller structure as well as offers some nice interpretations, which add some useful insight into properties of MLC-based thermal and SOC balancers. These particular aspects are analyzed and discussed in detail (second contribution). The sensitivity and robustness of the control scheme to parametric uncertainty and cell resistance variation with temperature is also analyzed (third contribution).
In addition, the control behavior and the effect of driving style and prediction horizon on the balancing performance are also critically analyzed through simulations (fourth contribution).

The proposed control scheme is thoroughly evaluated in a simulation study for various real world and certification drive cycles. The study is focussed on an air-cooled modular battery consisting of only four modules for illustration purpose. For simplicity, the electrical behavior of cells is described using simple cell model [24] based on resistive circuits, whereas thermal behavior is modeled using lumped capacitance and flow network modeling approach [11], [25]-[27]. In order to analyze the effectiveness of the control scheme, the cells are assumed to have significant differences in their resistances, capacities, and initial SOCs. The load on the modular battery is assumed to be three-phase electric drive of Toyota Prius PHEV running in pure EV mode.

The paper is organized as follows. Section II summarizes the notation used in this paper. Section III gives an overview of MLC-based modular battery. The electro-thermal state-space model and the original problem formulation are presented in sections IV and V respectively. The problem reformulation as LQ MPC scheme is presented in section VI. The controller interpretation and sensitivity analysis are presented in section VII. The simulation setup is presented in section VIII and the control simulation results and performance are discussed at length in section IX. Finally, section X concludes the paper.

\section{NOTATION}

Throughout this paper, we use $\mathbb{R}\left(\mathbb{R}_{+}\right), \mathbb{R}^{n}\left(\mathbb{R}_{+}^{n}\right)$, and $\mathbb{R}^{n \times m}$ to denote set of (non-negative) real numbers, set of real vectors with $n$ (non-negative) elements, and set of real matrices with order $n \times m$ respectively. Unless otherwise noted, we use calligraphic letters to denote subsets of real vector spaces. We use $I_{n}$ to denote the identity matrix of order $n \times n, 1_{n}$ to denote column $n$-vector of ones, $0_{n}$ to denote column $n$ vector of zeros. We use $\|\cdot\|$ and $|\cdot|$ to denote Euclidean norm and absolute value of variables respectively whereas $\|x\|_{Q}^{2}$ is used to denote $x^{\mathrm{T}} Q x$. For sake of saving space, we also occasionally use MATLAB's notation 'diag' and 'bl kdiag' to denote diagonal and block-diagonal matrices respectively and 'vertcat' to denote vertical concatenation.

\section{MLC-BASEd MOdUlar BATTERy: InTROdUCTION}

The (cascaded h-bridge) MLC-based modular battery, shown in Fig. 1, consists of $n$ series-connected power

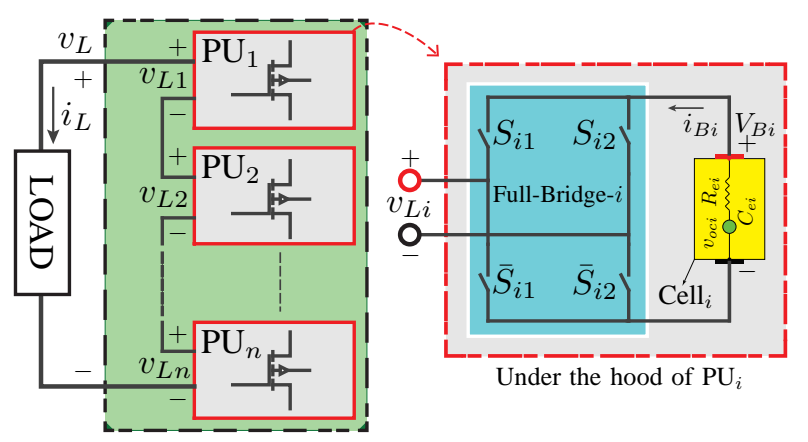

Fig. 1. Block diagram of an MLC-based modular battery inside green box. 
units (PUs), each containing a full-bridge (FB) and an isolated Cell $_{i}$. It supplies voltage $v_{L}(t)=\sum_{i=1}^{n} v_{L i}(t) \in$ $\left[0, v_{L, \max }\right] \subseteq \mathbb{R}_{+}$to a variable load with current demand $i_{L}(t) \in\left[i_{L, \min }, i_{L, \max }\right] \subseteq \mathbb{R}$, where $v_{L i}$ is the terminal voltage of $\mathrm{PU}_{i}$. Each $\mathrm{FB}_{i}$ can be operated in all four quadrants of the $i_{L}-v_{L i}$ plane using a switching function $s_{i}(t) \in\{-1,0,1\}$, which generates $v_{L i}(t) \in\left\{-V_{B i}, 0,+V_{B i}\right\}$ where $V_{B i}$ is cell terminal voltage. This enables control of bidirectional power flow from each $\mathrm{Cell}_{i}$, making it suitable for balancing purpose. In this paper, we are only interested in controlling the average behavior of the switched modular battery during each switching period $T_{\mathrm{sw}}$ of $s_{i}(t)$. Therefore, we define duty-cycle

$$
u_{i}(t):=\frac{1}{T_{\mathrm{sw}}} \int_{t-T_{\mathrm{sw}}}^{t} s_{i}(\tau) \mathrm{d} \tau
$$

for each $\mathrm{PU}_{i}$. Note that $u_{i}(t) \in[-1,1]$, where negative value implies $v_{L i}(t)<0$ and nonnegative value implies $v_{L i}(t) \geq 0$.

\section{A. Battery Control Modes}

The switch control framework based on the control variable $u_{i}$ allows two types of battery control modes, namely unipolar and bipolar control modes. The unipolar control mode $\left(u_{i}(t) \in[0,1]\right)$ does not allow polarity inversion of any cell in the string, which implies that at any time instant, either all cells are charging or all are discharging depending on the direction of $i_{L}(t)$ (i.e. $v_{L i}(t) v_{L j}(t) \geq 0$ ). On the other hand, the bipolar control mode $\left(u_{i}(t) \in[-1,1]\right)$ allows polarity inversion (i.e. $v_{L i}(t) v_{L j}(t)<0$ ) of some cells in the string, which implies that, at any time instant, it is possible to charge some cells while discharging others. The detailed comparison between unipolar and bipolar modes will be carried out in our future work. This study is restricted to unipolar mode.

\section{B. Terminal Behavior of $P U_{i}$}

The average signals on two ports of ideal $\mathrm{FB}_{i}$ (see Fig. 1) are linearly related through unipolar duty cycle $u_{i}$ as follows

$$
i_{B i}(t)=i_{L}(t) u_{i}(t), \quad v_{L i}(t)=d_{v i}(t) u_{i}(t),
$$

where $i_{L}$ and $v_{L i}$ are the terminal current and voltage of $\mathrm{PU}_{i}$ respectively, $i_{B i}$ is the current through $\mathrm{Cell}_{i}$, and

$$
d_{v i}(t)=v_{o c i}-i_{L}(t) R_{e i}
$$

is the ON-time terminal voltage of $\mathrm{Cell}_{i}$, where $v_{\text {oci }}$ is $\mathrm{OCV}$ and $R_{e i}$ is internal resistance. The terminal power of the modular battery is given by $P_{L}(t)=\sum_{i=1}^{n} P_{L i}(t)$, where $P_{L i}(t)=v_{L i}(t) i_{L}(t)$ is the terminal power of $\mathrm{PU}_{i}$. See [18] and [19] for detailed derivation of these averaged variables.

\section{State Space Electro-Thermal Model}

The state space electro-thermal model of an air-cooled modular battery with $n$ modules is presented on standard form in this section. The model, see [17]-[19] for its origin, is built based on the following assumptions.

- Load demand $\left(i_{L}(t), v_{L d}(t)\right)$ at current instant is given.

- The electrical model is based on the simple cell model (OCV-R i.e. resistance in series with OCV) [24].
- The OCV of all cells is assumed constant. This approximation is somewhat justified for some lithium batteries (like $\mathrm{LiFePO}_{4}$ /graphite (LFP)) if we assume battery operation in a typical SOC window of $20 \%$ to $90 \%$ [7].

- The thermal model is based on lumped capacitance and flow network modeling approach [11]. It considers only cell surface temperature. The coolant flow is assumed laminar with known constant inlet temperature and speed.

\section{A. Electrical Model}

The electrical model of each $\mathrm{PU}_{i}$ is given by

$$
\dot{\xi}_{i}(t)=-\frac{1}{3600 C_{e i}} i_{L}(t) u_{i}(t), \quad v_{L i}(t)=d_{v i}(t) u_{i}(t),
$$

where $\xi_{i}$ is the SOC and $C_{e i}$ is the coulomb capacity of $\mathrm{Cell}_{i}$, $v_{L i}$ is the terminal voltage of $\mathrm{PU}_{i}$ in response to cell current $i_{B i}, d_{v i}$ is defined in (3), and $i_{L} \in\left[i_{L, \min }, i_{L, \max }\right] \subseteq \mathbb{R}$ is the demanded battery load current. Now, using (4) as a basic building block, the electrical subsystem of an $n$-cell modular battery is given by the following standard state-space model

$$
\begin{aligned}
\dot{\xi}(t) & =A_{E} \xi(t)+B_{E} i_{L}(t) u(t), \\
v_{L}(t) & =\sum_{i=1}^{n} v_{L i}=\sum_{i=1}^{n} d_{v i}(t) u_{i}=D_{v}(t) u(t),
\end{aligned}
$$

where $\xi(t)=\left[\begin{array}{lll}\xi_{1} & \cdots & \xi_{n}\end{array}\right]^{\mathrm{T}} \in \mathbb{R}^{n}$ is a state vector of SOCs, $u(t)=\left[\begin{array}{lll}u_{1} & \cdots & u_{n}\end{array}\right]^{\mathrm{T}} \in \mathbb{R}^{n}$ is the control input to generate positive $v_{L i}: \mathrm{s}$, and $v_{L}(t) \in\left[0, v_{L, \max }(t)\right] \subseteq \mathbb{R}_{+}$is the total terminal voltage of the modular battery, where

$$
v_{L, \max }(t)=D_{v}(t) \cdot 1_{n}
$$

is a maximum voltage capacity of the modular battery and

$$
D_{v}(t)=\left[\begin{array}{lll}
d_{v 1}(t) & \cdots & d_{v n}(t)
\end{array}\right] \in \mathbb{R}^{1 \times n} .
$$

See appendix for definition of matrices $A_{E}$ and $B_{E}$.

\section{B. Thermal Model}

The surface temperature dynamics of each $\operatorname{Cell}_{i}$ is given by

$$
\dot{T}_{s i}(t)=\sum_{j=1}^{i} a_{t i j} T_{s j}(t)+\frac{R_{e i}}{C_{s i}} i_{L}^{2}(t) u_{i}(t)+w_{t i} T_{f 0},
$$

where $i_{L}^{2}(t) u_{i}(t), R_{e i}$, and $C_{s i}$ are the rms current during each switching period, the internal resistance, and the heat capacity of Cell $i_{i}$ and $T_{f 0}$ is the constant inlet coolant temperature (measured disturbance). The coefficients $a_{t i j}$, given by (A.51a), describes unidirectional thermal coupling from upstream Cell ${ }_{j}$ to downstream $\mathrm{Cell}_{i}$ due to convective heat transfer in the coolant, whereas $w_{t i}$, given by (A.51b), describes the influence of $T_{f 0}$ on $\mathrm{Cell}_{i}$. Now, using (8), the thermal subsystem of $n$ cell modular battery is given by

$$
\dot{T}_{s}(t)=A_{T} T_{s}(t)+B_{T} i_{L}^{2}(t) u(t)+W_{T} T_{f 0},
$$

where $T_{s}=\left[\begin{array}{lll}T_{s 1} & \cdots & T_{s n}\end{array}\right]^{\mathrm{T}} \in \mathbb{R}^{n}$ is the thermal state vector, and $W_{T}=\left[\begin{array}{lll}w_{t 1} & \cdots & w_{t n}\end{array}\right]^{\mathrm{T}} \in \mathbb{R}^{n}$ is a $T_{f 0}$ influence vector. For a control constrained LQ problem formulation, we rewrite (9) on the following standard state-space form

$$
\dot{\vartheta}(t)=A_{\vartheta} \vartheta(t)+B_{\vartheta} i_{L}^{2}(t) u(t)
$$


TABLE I

DEFINITION OF CELl AND COOLANT PARAMETERS

\begin{tabular}{|c|c|c|}
\hline Parameters & Expression & Units \\
\hline $\begin{array}{l}\text { OCV of Cell } \\
\text { Electrical Resistance of Cell } \\
\text { Charge Capacity of Cell } \\
\text { Mass of Cell } \\
\text { Meat Capacity of Cell } \\
\text { Heat } \\
\text { Thermal Resistance of Cell } \\
\text { Air Volumetric Flow Rate } \\
\text { Air Thermal Conductance } \\
\text { Temperature Coefficients } \\
\text { Thermal Coupling Coefficients } \\
\text { Thermal Coupling Coefficients }\end{array}$ & $\begin{array}{c}v_{o c i} \\
R_{e i} \\
C_{e i} \\
m_{i} \\
C_{s i}=\rho_{s i} c_{p s i} V_{s i} \\
R_{u i} \\
\dot{V}_{f} \\
c_{f} \\
a_{s i}=\left(C_{s i} R_{u i}\right)^{-1} \\
\alpha_{i}=R_{u i} c_{f} \\
\beta_{i}=-1+\alpha_{i}\end{array}$ & $\begin{array}{c}V \\
\Omega \\
A h \\
k g \\
J K^{-1} \\
K W^{-1} \\
m^{3} s^{-1} \\
W K^{-1} \\
s^{-1} \\
\text { Unitless } \\
\text { Unitless }\end{array}$ \\
\hline
\end{tabular}

in terms of augmented state $\vartheta(t)=\left[\begin{array}{ll}T_{s}^{\mathrm{T}}(t) & T_{f 0}\end{array}\right]^{\mathrm{T}} \in \mathbb{R}^{n+1}$, where $T_{f 0}$ is treated as a dummy state. See appendix for definitions of matrices $A_{T}, B_{T}, A_{\vartheta}$, and $B_{\vartheta}$ and Table $\mathrm{I}$ for definitions of various cell and coolant parameters.

Remark 1. The cell resistance varies slowly with temperature in normal operating range $[25,40]^{\circ} \mathrm{C}$. Therefore, we can assume cell resistance to be constant, during each small sampling interval, for control design. The resistance variation over large temperature range can be compensated using gainscheduling at much slower rate. See section VII-B.

\section{Complete State-Space Model}

The complete continuous-time electro-thermal model of an $n$-cell modular battery is given by the following standard linear time-varying (LTV) state-space system

$$
\begin{aligned}
& \dot{x}(t)=A x(t)+B\left(i_{L}(t)\right) u(t), \\
& y(t)=C x(t)+D\left(i_{L}(t)\right) u(t),
\end{aligned}
$$

where $x(t)=\left[\begin{array}{lll}\xi^{\mathrm{T}}(t) & \vartheta^{\mathrm{T}}(t)\end{array}\right]^{\mathrm{T}} \in \mathbb{R}^{2 n+1}$ is the state vector, $y(t)=\left[\begin{array}{ll}\vartheta^{\mathrm{T}}(t) & v_{L}(t)\end{array}\right]^{\mathrm{T}} \in \mathbb{R}^{n+2}$ is the output vector. All state-space matrices are defined in appendix.

\section{Model Discretization}

The discrete-time state-space model is given by

$$
\begin{aligned}
x(k+1) & =A_{d} x(k)+B_{d}\left(i_{L}(k)\right) u(k), \\
y(k) & =C x(k)+D\left(i_{L}(k)\right) u(k),
\end{aligned}
$$

where matrices $A_{d}$ and $B_{d}$ are obtained using Euler approximation assuming $i_{L}(k)$ to be constant during each sampling interval $[k h,(k+1) h)$ where $h$ is a sampling step size.

\section{E. SOC and Capacity Under Parametric Variations}

In this study, we assume differences in cell capacities, initial SOC levels, and resistances. These variations may result in cell imbalances i.e. imbalance among cell temperatures as well as dischargeable and chargeable capacities of cells given by

$$
C_{e d, i}(k)=\xi_{i}(k) C_{e i}, \quad C_{e c, i}(k)=\left(1-\xi_{i}(k)\right) C_{e i} .
$$

Under these imbalances, the effective capacity and SOC of the battery pack are respectively given by

$$
C_{\mathrm{B}}(k)=C_{\mathrm{B}, d}(k)+C_{\mathrm{B}, c}(k), \quad \xi_{\mathrm{B}}(k)=\frac{C_{\mathrm{B}, d}(k)}{C_{\mathrm{B}}(k)},
$$

where $C_{\mathrm{B}, d}(k)=\xi_{\mathrm{B}}(k) C_{\mathrm{B}}(k)=\min _{i}\left(C_{e d, i}(k)\right)$ and $C_{\mathrm{B}, c}(k)=\left(1-\xi_{\mathrm{B}}(k)\right) C_{\mathrm{B}}(k)=\min _{i}\left(C_{e c, i}(k)\right)$ are remaining dischargeable and chargeable capacities of the battery respectively. The main purpose of SOC balancer is to maximize $C_{\mathrm{B}}(k)$, which is maximized if SOC of all cells are equalized.

\section{Convex Problem Formulation Using FULL FUTURE LOAD INFORMATION}

The control objectives include thermal and SOC balancing and terminal voltage control of the modular battery. In this section, the control problem is formulated for a case where full driving information is accessible. In particular, as an extension of our earlier studies [17]-[19], the problem formulation is formalized here for standard LQ control design in section VI. In addition, the design of objective function is properly motivated and the constraints are formulated as polyhedra to get optimization problem on standard QP form.

\section{A. SOC and Temperature Deviations/Balancing Errors}

Let us define SOC and temperature error vectors

$$
\begin{aligned}
e_{\xi}(k) & =\xi(k)-\bar{\xi}(k) \cdot 1_{n}=M_{e} \xi(k), \\
e_{T_{s}}(k) & =T_{s}(k)-\bar{T}_{s}(k) \cdot 1_{n}=M_{e} T_{s}(k),
\end{aligned}
$$

where $\bar{\xi}(k)=\frac{1}{n} 1_{n}^{\mathrm{T}} \xi(k)$ and $\bar{T}_{s}(k)=\frac{1}{n} 1_{n}^{\mathrm{T}} T_{s}(k)$ are instantaneous mean SOC and mean temperature of the modular battery and can be considered as reference signals here. The matrix

$$
M_{e}=\left(I_{n}-\frac{1}{n} 1_{n \times n}\right) \in \mathbb{R}^{n \times n},
$$

maps each state vector to its corresponding error vector.

\section{B. Design of Objective Function}

The SOC balancing is primarily needed to keep cells balanced at least at SOC-boundaries (i.e. end-of-charge (EOC) and end-of-discharge (EOD) states) during charge/discharge cycling of the battery to avoid non-uniform ageing [6]. In other words, a temporary SOC imbalance during cycling may be allowed if cells are balanced at EOC and EOD. However, in vehicle applications, lower SOC boundary (terminal time of journey) is normally not fixed due to uncertainty in a drive cycle. Therefore, we propose to reduce SOC deviations as much as possible during cycling to get fairly balanced DODs by the end of a driving trip. Similarly, we propose to minimize temperature deviations all the time during cycling because cell ageing is exponential in cell temperature [5]. These minimizations must be achieved without increasing average battery temperature relative to that of unbalanced battery and by using minimum possible actuation of cells. These objectives are formulated as following cost functions

$$
\begin{aligned}
J_{E} & =\sum_{k=0}^{N-1}\left\|e_{\xi}(k)\right\|_{Q_{E}}^{2}+\left\|e_{\xi}(N)\right\|_{P_{E}}^{2}, \\
J_{T} & =\sum_{k=0}^{N-1}\left\|e_{T_{s}}(k)\right\|_{Q_{T}}^{2}+\left\|e_{T_{s}}(N)\right\|_{P_{T}}^{2}, \\
J_{\bar{T}} & =\sum_{k=0}^{N-1}\left\|\bar{T}_{s}(k)\right\|_{q_{\bar{t}}}^{2}+\left\|\bar{T}_{s}(N)\right\|_{p_{\bar{t}}}^{2}, \\
J_{u} & =\sum_{k=0}^{N-1}\|u(k)\|_{R_{u}}^{2},
\end{aligned}
$$


where $N$ is prediction horizon, $Q_{E}=q_{e} I_{n}, P_{E}=p_{e} I_{n}, Q_{T}=$ $q_{t} I_{n}$, and $P_{T}=p_{t} I_{n}$ are positive semidefinite penalty matrices for deviations, $q_{e}, p_{e}, q_{t}, p_{t}, q_{\bar{t}}$, and $p_{\bar{t}}$ are nonnegative scalar penalty weights, and $R_{u}=r_{u} I_{n}$ is a positive definite penalty matrix for control with weight $r_{u}>0$. There are multiple objectives and it is impossible to minimize them individually. Therefore, a scalar objective function is formulated as follows

$$
J=\gamma_{1} J_{E}+\gamma_{2} J_{T}+\gamma_{3} J_{\bar{T}}+\gamma_{4} J_{u},
$$

where $\gamma_{i} \geq 0$ are trade-off weights, which signify the relative importance of each objective. These weights are chosen, such that $\sum_{i}^{4} \gamma_{i}=1$, to achieve desired trade-off between temperature and SOC balancing performance.

Objective Function on Standard Form: The objective function (19) is in quadratic form in terms of state errors whereas system dynamics is written in terms of states. Therefore, transformations (15) and (16) are applied to get the following standard quadratic objective function

$$
J=\sum_{k=0}^{N-1}\left[\|x(k)\|_{\bar{Q}_{x}}^{2}+\gamma_{4}\|u(k)\|_{R_{u}}^{2}\right]+\|x(N)\|_{\bar{P}_{x}}^{2},
$$

in terms of $x(k)$ and $u(k)$, where

$$
\begin{aligned}
\bar{Q}_{x} & =\operatorname{blkdiag}\left(\gamma_{1} \bar{Q}_{E}, \gamma_{2} \bar{Q}_{T}+\gamma_{3} \bar{Q}_{\bar{T}}, 0\right), \\
\bar{P}_{x} & =\operatorname{blkdiag}\left(\gamma_{1} \bar{P}_{E}, \gamma_{2} \bar{P}_{T}+\gamma_{3} \bar{P}_{\bar{T}}, 0\right),
\end{aligned}
$$

are new penalty weight matrices with

$$
\begin{array}{ll}
\bar{Q}_{E}=M_{e}^{\mathrm{T}} Q_{E} M_{e}, \bar{P}_{E}=M_{e}^{\mathrm{T}} P_{E} M_{e}, \\
\bar{Q}_{T}=M_{e}^{\mathrm{T}} Q_{T} M_{e}, & \bar{P}_{T}=M_{e}^{\mathrm{T}} P_{T} M_{e}, \\
\bar{Q}_{\bar{T}}=\frac{q_{\bar{t}}}{n^{2}} 1_{n \times n}, & \bar{P}_{\bar{T}}=\frac{p_{\bar{t}}}{n^{2}} 1_{n \times n},
\end{array}
$$

where the matrix $M_{e}$ is defined in (17).

\section{State Constraints}

Running State Constraints: The balancer must ensure the following constraints during driving

$$
\begin{gathered}
\xi_{\min } \leq \xi(k) \leq \xi_{\max }, \quad \forall k \\
\left|e_{\xi_{i}}(k)\right| \leq \delta \xi, \quad \forall i, \quad \forall k \geq k_{b}, \\
T_{s, \min } \leq T_{s}(k) \leq T_{s, \max }, \quad \forall k \\
\left|e_{T_{s i}}(k)\right| \leq \delta T_{s}, \quad \forall i, \quad \forall k
\end{gathered}
$$

for SOC and thermal balancing, where vectors $\xi_{\min } / T_{s, \min }$ and $\xi_{\max } / T_{s, \max }$ give minimum and maximum limits of SOC/temperature for each cell, scalars $\delta \xi$ and $\delta T_{s}$ are $S O C$ and thermal deviation allowances for all cells, and $k_{b}$ is an initial balancing time period. From constraints (24a), the initial set of SOCs can be defined as following polyhedron

$$
\mathcal{X}_{E 0}=\left\{\xi \mid H_{E 0} \xi \leq h_{e 0}\right\},
$$

for suitably defined constraint matrix $H_{E 0}$ and vector $h_{e 0}$. Similarly, by first applying transformations (15) and (16) to (24b) and (24d) respectively and then using (24a)-(24b) and $(24 c)-(24 d)$, the goal/target sets of SOCs and temperatures can be easily defined as following polyhedra

$$
\mathcal{X}_{E g}=\left\{\xi \mid H_{E g} \xi \leq h_{e g}\right\}, \mathcal{X}_{T g}=\left\{T_{s} \mid H_{T g} T_{s} \leq h_{t g}\right\},
$$

for suitably defined constraint matrices $H_{E g}, H_{T g}$ and vectors $h_{e g}, h_{t g}$. The primary goal of thermal and SOC balancing is to first drive initial SOC of all cells to $\mathcal{X}_{E g}$ within a certain balancing time $t_{b}=h k_{b}$ and then keep them there, whereas temperature of all cells must stay within $\mathcal{X}_{T g}$ all the time.

Terminal State Constraint: If a drive cycle is known a priori then the balancer may also achieve a secondary goal i.e. perfect SOC equalization by the end of driving trip. This can be specified as the following terminal constraint

$$
\xi_{i}\left(N_{d}\right)=\xi_{j}\left(N_{d}\right), \forall i, j
$$

\section{Output Voltage Tracking Constraint}

In addition to balancing, the battery must strictly satisfy the load voltage demand $v_{L d}(k)$. Using voltage equation (5b), this is modeled as the following voltage tracking constraint

$$
v_{L}(k)=D_{v}(k) u(k)=v_{L d}(k)
$$

where $D_{v}(k)$ is defined in equation (7).

\section{E. Control Constraint Set}

The unipolar control mode imposes control constraint $u_{i}(k) \in[0,1]$ for each Cell $_{i}$. Therefore, the control constraint set of $n$-cell modular battery is given by

$$
u(k) \in \mathcal{U}=\left\{u(k) \mid H_{u} u \leq h_{u}, \forall k\right\},
$$

where $H_{u}=\operatorname{vertcat}\left(I_{n},-I_{n}\right)$ and $h_{u}=\operatorname{vertcat}\left(1_{n}, 0_{n}\right)$. Note that $\mathcal{U}$ is a convex set (hypercube in $\mathbb{R}_{+}^{n}$ ).

\section{F. Constrained Convex Optimization Problem}

If future load demand $\left(i_{L}(k), v_{L d}(k)\right)$ over the whole driving cycle of length $N_{d}$ is fully accessible then the electrothermal control problem can be formulated as the following state and control constrained convex optimization problem.

$$
\begin{aligned}
& \operatorname{minimize} J\left(x(0), u\left(0: N_{d}-1\right)\right) \\
& \text { subject to } \\
& x(k+1)=A_{d} x(k)+B_{d}(k) u(k), \\
& D_{v}(k) u(k)=v_{L d}(k), \\
& \xi(k) \in \mathcal{X}_{E g}, \forall k \geq k_{b}, T_{s}(k) \in \mathcal{X}_{T g} \\
& \xi_{i}\left(N_{d}\right)=\xi_{j}\left(N_{d}\right), \forall i, j \\
& u(k) \in \mathcal{U},
\end{aligned}
$$

with optimization variables $x(k)$ and $u(k)$ for all $k \in$ $\left\{0, \cdots, N_{d}-1\right\}$ where objective function $J$ is defined in (20). Note that the initial balancing time period $k_{b}$ is not fixed as it depends on characteristics of battery load profile. To make the problem independent of $k_{b}$, we can soften state inequality constraints using a slack variable approach [23]. The problem (P-I) can be solved offline using CVX, which is a MATLAB-based package for specifying and solving convex programs using disciplined convex programming ruleset [28][30]. The solution is globally optimal as it is based on full future driving information. A variant of this problem, although not on standard form, is solved in our earlier studies [17]-[19]. 


\section{Short LoAd Predictions: LQ MPC Scheme With ORTHOGONAL CONTROL DECOMPOSITION}

If full future driving information is not accessible then (P-I) cannot be solved. Therefore, we propose an alternative control algorithm based on LQ MPC scheme to solve the original problem using limited future driving information (i.e. $N \ll$ $N_{d}$ ). The scheme is developed with the following aims.

- The load voltage regulation is prioritized and thermal and SOC balancing are achieved as secondary objectives.

- To secure feasibility, we aim to achieve balancing objectives without imposing any hard state constraints.

- The voltage constraint in the original problem poses an issue for transforming the overall problem to standard LQ form. This issue is addressed in a special way by separating voltage control task as described below.

Keeping in view all the above aims, we propose to decompose the control problem into two cascaded subproblems:

1) Voltage Controller $\left[u_{v}(k)\right]$ : It is a feedforward controller, which uses information only about load demand $v_{L d}(k)$ and $i_{L}(k)$ given at each time instant, to generate $v_{L}(k)=$ $v_{L d}(k)$. The control signal $u_{v}(k)$ is computed analytically (minimum norm solution), see section VI-B.

2) Balancing Controller $\left[u_{b}(k)\right]$ : It is a feedback controller cascaded with outer voltage controller. It uses information about battery state $x(k), i_{L}(k)$, and $u_{v}(k)$, to achieve thermal and SOC balancing. We propose to choose the optimal control decision $u_{b}(k)$ such that it is always orthogonal to $u_{v}(k)$. This guarantees the voltage constraint satisfaction while giving the possibility of simultaneous balancing. The balancing control $u_{b}(k)$ is computed in receding horizon fashion based on control-constrained $L Q$ problem (LQ MPC problem), see section VI-C.

This novel approach of separating balancing controller from voltage controller offers multiple advantages. Firstly, it facilitates the formulation of the electro-thermal control problem on standard LQ MPC form. Secondly, it allows precomputation of voltage control decisions for a pre-selected grid of demanded load currents and voltages. Thirdly, it gives us more insight into structural and functional properties of the controller. All the important ingredients of the proposed control scheme are presented below in detail.

\section{A. Orthogonal Control Decomposition}

Let us consider the voltage constraint $\left(D_{v}(k) u(k)=\right.$ $\left.v_{L d}(k)\right)$ in the optimization problem (P-I). This single output equality constraint can be satisfied using $n$ control variables. Hence, there are multiple solutions and the nullspace of $D_{v}$ provides $n-1$ degrees-of-freedom in generating $v_{L}$. This extra freedom can be used for the balancing objectives.

The main idea is to decompose the control signal into two orthogonal components, one for voltage control and other for balancing control. Using decomposition theorem of linear algebra [31, Theorem 3.14], we propose the following unique decomposition of total control vector $u \in \mathcal{U} \subseteq \mathbb{R}^{n}$

$$
\begin{aligned}
& u(k)=u_{v}(k)+u_{b}(k), \\
& u_{v}(k) \in \mathcal{N}\left(D_{v}(k)\right)^{\perp}, \quad u_{b}(k) \in \mathcal{N}\left(D_{v}(k)\right),
\end{aligned}
$$

where $\mathcal{N}\left(D_{v}(k)\right)$ is the time-varying nullspace of $D_{v}(k)$ and $\mathcal{N}\left(D_{v}(k)\right)^{\perp}$ is the orthogonal complement of $\mathcal{N}\left(D_{v}(k)\right)$. The nullspace is a hyperplane in $\mathbb{R}^{n}$ given by

$$
\mathcal{N}\left(D_{v}\right)=\left\{u(k) \mid D_{v}(k) u(k)=0\right\}=\mathcal{R}\left(V_{\mathrm{n}}\right) \subseteq \mathbb{R}^{n},
$$

where $\mathcal{R}\left(V_{\mathrm{n}}\right)$ is the range-space of null-space basis matrix

$$
V_{\mathrm{n}}(k)=\left[\begin{array}{lll}
v_{\mathrm{n}, 1}(k) & \cdots & v_{\mathrm{n}, n-1}(k)
\end{array}\right] \in \mathbb{R}^{n \times n-1},
$$

which contains parameterized orthonormal basis vectors $v_{\mathrm{n}, i}(k) \in \mathbb{R}^{n}$ of null-space. The basis $V_{\mathrm{n}}(k)$ of nullspace is not unique. A particular choice, obtained using MATLAB ${ }^{\circledR}$ Symbolic Toolbox, is given by

$$
V_{\mathrm{n}}(k)=\left[\begin{array}{c}
V_{\mathrm{n}}^{\prime}(k) \\
I_{n-1}
\end{array}\right],
$$

where $V_{\mathrm{n}}^{\prime}(k)=-\left[\begin{array}{lll}\frac{d_{v 2}(k)}{d_{v 1}(k)} & \cdots & \frac{d_{v n}(k)}{d_{v 1}(k)}\end{array}\right] \in \mathbb{R}^{1 \times(n-1)}$, is a row vector with elements $d_{v i}(k)=v_{o c i}-i_{L}(k) R_{e i}$. The orthogonal complement of nullspace is given by

$$
\mathcal{N}\left(D_{v}\right)^{\perp}=\mathcal{R}\left(D_{v}^{\mathrm{T}}\right)=\left\{u(k) \mid u(k)=\alpha_{v}(k) D_{v}(k)^{\mathrm{T}}\right\},
$$

where $\alpha_{v}(k)$ is a scalar design parameter. Now we are ready to design $u_{v}(k)$ and $u_{b}(k)$ constrained inside time-varying orthogonal subspaces $\mathcal{R}\left(D_{v}(k)^{\mathrm{T}}\right)$ and $\mathcal{N}\left(D_{v}(k)\right)$ respectively.

\section{B. Voltage Controller}

According to (33), $u_{v}(k) \in \mathcal{R}\left(D_{v}(k)^{\mathrm{T}}\right)$ is given by

$$
u_{v}(k)=\alpha_{v}(k) D_{v}(k)^{\mathrm{T}} .
$$

To generate voltage $D_{v}(k) u_{v}(k)=v_{L d}(k)$, we simply choose

$$
\alpha_{v}(k)=\frac{v_{L d}(k)}{\left\|D_{v}(k)\right\|^{2}} .
$$

With this particular choice of $\alpha_{v}(k),(34)$ can be rewritten as

$$
u_{v}(k)=D_{v}^{\dagger}(k) v_{L d}(k)
$$

where $D_{v}^{\dagger}(k)=D_{v}(k)^{\mathrm{T}}\left(D_{v}(k) D_{v}(k)^{\mathrm{T}}\right)^{-1}=\frac{D_{v}(k)^{\mathrm{T}}}{\left\|D_{v}(k)\right\|^{2}}$, is a right pseduo-inverse of $D_{v}(k)$. Now it is clear that (36) gives a minimum norm solution. Note that $D_{v}^{\dagger}(k)$ will always be a positive column vector for a given positive row vector $D_{v}(k)$. This is indeed a case in our model provided that $i_{L}(k)$ stays within absolute maximum current rating of a cell. Therefore, the analytical solution (36) will always result in $u_{v}(k) \geq 0_{n}$ for $v_{L d} \geq 0$ and $i_{L}(t) \in\left[i_{L, \min }, i_{L, \max }\right]$. Moreover, $u_{v}(k)<1_{n}$ is guaranteed if the demanded voltage satisfies the constraint $v_{L d}(k) \in\left[0, v_{L d, \max }\right]$ with

$$
v_{L d, \max }<v_{L, \max }(k), \quad \forall k
$$

where $v_{L, \max }(k)$, defined in (6), is the maximum voltage capacity of the modular battery. Therefore, the solution $u_{v}(k) \in$ $\mathcal{R}\left(D_{v}(k)^{\mathrm{T}}\right)$ given by (36) is guaranteed to be inside $\mathcal{U}$ as well at any time instant under the stated conditions. 


\section{Balancing Controller: LQ MPC Problem}

After computing the voltage control $u_{v}$, the balancing control $u_{b} \in \mathcal{N}\left(D_{v}\right)$ can be chosen using MPC scheme to achieve thermal and SOC balancing objectives. Before presenting this scheme, let us first represent the balancing control as follows

$$
u_{b}(k)=\sum_{i=1}^{n-1} \rho_{b i}(k) v_{\mathrm{n}, i}(k)=V_{\mathrm{n}}(k) \rho_{b}(k) \in \mathcal{U}_{b}
$$

where $V_{\mathrm{n}}(k)$ is given by (32) and $\rho_{b}(k) \in \mathbb{R}^{n-1}$ are coefficients of null-space basis vectors, and

$$
\mathcal{U}_{b}(k)=\left\{u_{b}(k)=V_{\mathrm{n}}(k) \rho_{b}(k) \mid\left(u_{v}(k)+u_{b}(k)\right) \in \mathcal{U}\right\},
$$

is a time-varying set of feasible $u_{b}(k)$ whose boundaries vary with load demand $\left(i_{L}, v_{L d}\right)$ as well as cell resistances and OCVs. Note that using $\rho_{b}$ as a control variable reduces optimal decision search from $\mathbb{R}^{n}$ to $\mathbb{R}^{n-1}$. It is equal to last $n-1$ elements of $u_{b}$ for the particular choice of $V_{\mathrm{n}}$ given by (32).

Constrained LQ MPC Standard Form: The control $\rho_{b}(k)$ is computed by solving a constrained LQ problem in a receding horizon fashion. Before presenting the problem, let us substitute $u(k)$ with $u_{b}(k)=V_{\mathrm{n}}(k) \rho_{b}(k)$ in (12a) and (20), to get the system dynamics and the objective function on the following standard forms in terms of $x(k)$ and $\rho_{b}(k)$

$$
\begin{gathered}
x(k+1)=A_{d} x(k)+\bar{B}_{d}(k) \rho_{b}(k), \\
J=\sum_{k=0}^{N-1}\left[\|x(k)\|_{\bar{Q}_{x}}^{2}+\left\|\rho_{b}(k)\right\|_{R_{\rho_{b}}}^{2}\right]+\|x(N)\|_{\bar{P}_{x}}^{2},
\end{gathered}
$$

where $R_{\rho_{b}}(k)=\gamma_{4} V_{n}^{\mathrm{T}}\left(i_{L}(k)\right) R_{u_{b}} V_{\mathrm{n}}\left(i_{L}(k)\right)$ is a time-varying penalty weight for $\rho_{b}(k)$ and $\bar{B}_{d}(k)=B_{d}(k) V_{\mathrm{n}}(k)$. Now using (40) and (41), the balancing control problem can be easily formulated on the following standard control-constrained $L Q$ form, which is solved to find the balancing control $u_{b}(k)$ at each time step $k \in\left\{0, \cdots, N_{d}-1\right\}$ in the MPC framework.

$$
\begin{aligned}
& \operatorname{minimize} J\left(x(k), \rho_{b}(k: k+N-1)\right) \\
& \text { subject to } \\
& x(k+l+1)=A_{d} x(k+l)+\bar{B}_{d}(k+l) \rho_{b}(k+l), \\
& u_{b}(k+l)=V_{\mathrm{n}}\left(\hat{i}_{L}(k+l)\right) \rho_{b}(k+l) \in \mathcal{U}_{b}(k+l), \\
& \forall l=\{0, \cdots, N-1\},
\end{aligned}
$$

with optimization variables $x(k+l)$ and $\rho_{b}(k+l)$ where $l$ is the time index for the prediction phase of MPC, $N_{d}$ is the driving horizon, $N \ll N_{d}$ is the prediction horizon, and $\mathcal{U}_{b}$, defined in (39), is a time-varying balancing control constraint set. The voltage control $u_{v}(k+l)$ needed for solving the problem (P-IV) is computed using analytical solution (36). Note that $\hat{i}_{L}(k+l)$ is the prediction of future load current demand. In this study, the full state and the load current are assumed to be perfectly available at current time step $k$. Therefore, the information needed to solve the problem is completely accessible if 1-step ahead prediction $(N=1)$ is used. For $N>1$ case, this study assumes that the load predictions $\hat{i}_{L}$ over a short horizon are perfectly known.

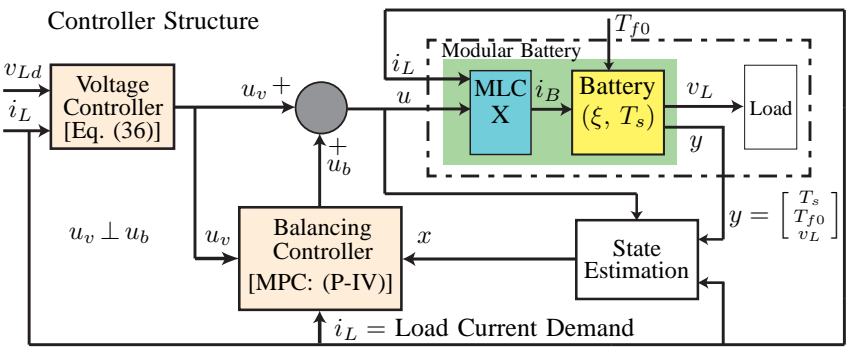

Fig. 2. Block diagram of closed-loop control system of MLC-based modular battery powering a variable load. This study assumes that full state is available.

\section{Summary of Electro-thermal Control Scheme}

The proposed control scheme for a given demand $\left(i_{L}, v_{L d}\right)$ and state $x(k)$ consists of following steps at each instant $k$ :

1. Find the voltage control $u_{v}(k)$ using (36) for given demand.

2. Solve the constrained LQ problem (P-IV) over a short prediction horizon $N \ll N_{d}$ to find the optimal control $u_{b}(k)=V_{\mathrm{n}}(k) \rho_{b}(k)$ for thermal and SOC balancing.

3. Compute complete controller using $u(k)=u_{v}(k)+u_{b}(k)$.

4. Apply the control input $u(k)$ to the system.

5. Let $k:=k+1$ and repeat steps $1-4$.

\section{CONTROLLER ANALYSIS AND INTERPRETATIONS}

\section{A. Controller Structure and Interpretation}

A block diagram of the battery electro-thermal control system, using voltage controller (36) and balancing controller (P-IV), is shown in Fig. 2. The total control $u(k)$ and the voltage control $u_{v}$ are both positively constrained. However, the balancing control $u_{b}$ may attain both positive and negative values. It can be interpreted as a control correction term with a possibility of both positive and negative corrections. The voltage controller first decides the duty of each cell to exactly satisfy the voltage demand without caring about balancing. The balancing controller then corrects (either increase or decrease) the duty of each cell in accordance with its balancing errors $e_{\xi_{i}}$ and $e_{T_{s i}}$ without disturbing the voltage. Note that the balancing control constraint set $\mathcal{U}_{b}(k)$, defined in (39), may be asymmetric about origin depending on the value of $u_{v}$.

The balancing control law in general is a piecewise-affine feedback in $x$. However, when constraints are inactive, it has a simple linear time-varying feedback structure given by

$$
u_{b}^{u}(k)=K_{u_{b}}^{e}\left(i_{L}(k)\right) \xi(k)+K_{u_{b}}^{\vartheta}\left(i_{L}(k)\right) \vartheta(k),
$$

where $K_{u_{b}}^{e}$ is a SOC control gain matrix and $K_{u_{b}}^{\vartheta}=$ $\left[\begin{array}{ll}K_{u_{b}}^{t} & K_{u_{b}}^{f}\end{array}\right]$ is a thermal control gain matrix where $K_{u_{b}}^{t}$ is a gain matrix for temperature control and $K_{u_{b}}^{f}$ is a gain vector for compensation of inlet fluid temperature. The gain matrices $K_{u_{b}}^{e}$ and $K_{u_{b}}^{\vartheta}$ have a special structure, which we will investigate in a separate study.

\section{B. Sensitivity Analysis}

The optimal control design presented in sections $\mathrm{V}$ and VI considers the affect of electrical actuation on temperature of cells. However, the effect of temperature on the electrical 


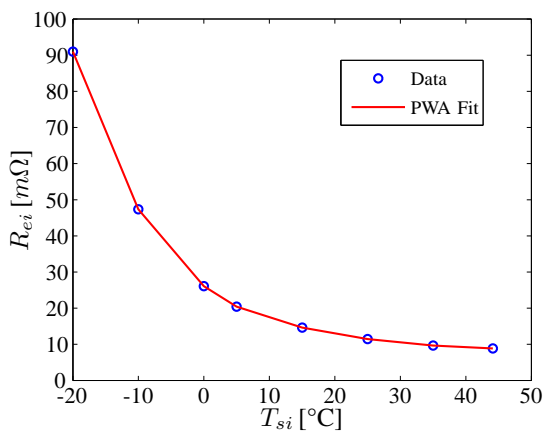

Fig. 3. Cell resistance variation as a function of temperature.

parameters of cells is neglected i.e. parameters are constant. The impact of this approximation is analyzed below.

An experimentally validated model for LFP cell is available in the literature, see [25]-[27]. According to these studies, the cell capacity and OCV have typically no short-term temperature dependence. However, the cell resistance (consequently the terminal voltage) varies as a function of temperature as shown in Fig. 3. We identified this curve through piecewise affine (PWA) approximation between consecutive data points obtained from the experimentally validated model. The figure shows that the resistance varies slightly with temperature in normal operating range $[25,40]^{\circ} \mathrm{C}$. Therefore, the controller can be designed assuming cell resistance to be constant for small temperature variation. The balancing controller uses state feedback and its objective is not to achieve zero steadystate errors. Therefore, it is inherently robust to small resistance variation as shown through simulations in section IX-D. The variation in resistances over large temperature range can be compensated using gain-scheduling at much slower rate.

However, the voltage controller uses only feedforward information to achieve voltage tracking. Therefore, the sensitivity of terminal voltage $v_{L}$ to small resistance variation around its nominal value is analyzed as follows. Let us first rewrite

$$
v_{L}=D_{v} u=v_{o c}^{\mathrm{T}} u-i_{L} R_{e}^{\mathrm{T}} u,
$$

where $v_{o c}=\left[v_{o c i}\right] \in \mathbb{R}^{n}$ and $R_{e}=\left[R_{e i}\right] \in \mathbb{R}^{n}$ are cell OCV and resistance vectors. Defining $R_{e B}=R_{e}^{\mathrm{T}} u \in \mathbb{R}_{+}$as total instantaneous resistance of modular battery, it follows

$$
v_{L}=v_{o c}^{\mathrm{T}} u-i_{L} R_{e B} \Rightarrow \partial v_{L}=-i_{L} \partial R_{e B} .
$$

For a meaningful relation, let us define $\partial R_{e B}=p_{R} R_{e B}^{\star}$ and $\partial v_{L}=p_{v} v_{L}^{\star}$, where $R_{e B}^{\star}$ and $v_{L}^{\star}=v_{L d}$ are nominal battery resistance and voltage, and $p_{R}$ and $p_{v}$ are relative/percentage variations in battery resistance and voltage. Now we can write

$$
p_{v}=-\left(\frac{i_{L} R_{e B}^{\star}}{v_{L d}}\right) p_{R}=-\left(\frac{V_{B \ell}^{\star}}{v_{L d}}\right) p_{R},
$$

where $V_{B \ell}^{\star}=i_{L} R_{e B}^{\star}$ is the nominal battery voltage loss. Now using $\left(1-\eta_{B}\right) \approx V_{B \ell}^{\star} / v_{L d}$ as a sensitivity function, it follows

$$
p_{v} \approx-\left(1-\eta_{B}\right) p_{R}
$$

where $\eta_{B}$ is the battery efficiency. Since $\eta_{B} \geq 0.9$ normally, we have $p_{v} \approx-0.1 p_{R}$. This simple analysis predicts very small error in $v_{L}$ for small variation in $R_{e B}$, which is also verified through simulations in Section IX-D.

\section{Power Flow under Balancing Controller}

The net power flow (so-called balancing power) through modular battery under $u_{b}$ is zero at each time instant as shown below (using the fact that the battery terminal voltage under balancing control is zero i.e. $v_{L b}=D_{v} \cdot u_{b}=0$, see (31)).

$$
P_{L b}(k)=i_{L}(k) v_{L b}(k)=i_{L}(k) D_{v}(k) u_{b}(k)=0 .
$$

Since each h-bridge is assumed as a lossless switch network, the total balancing power of all cells is also zero i.e.

$$
P_{L b}(k)=P_{B b}(k)=\sum_{i=1}^{n} P_{B b, i}(k)=0
$$

where $P_{B b, i}(k)=P_{L b, i}(k)=d_{v i}(k) i_{B b, i}(k)$ is the balancing power of each cell. Now using $i_{B b, i}(k)=i_{L}(k) u_{b i}(k)$ and substituting $d_{v i}(k)$ with (3), we get

$$
P_{B b}(k)=P_{B b, g}(k)-P_{B b, \ell}(k)=0
$$

where $P_{B b, g}(k)=i_{L}(k) \sum_{i=1}^{n} v_{o c i} u_{b i}(k)$ is the total internal balancing power generated by total OCV source and $P_{B b, \ell}(k)=i_{L}^{2}(k) \sum_{i=1}^{n} R_{e i} u_{b i}(k)$ is the total internal battery balancing power loss. The equation (47) shows that the total balancing power is zero, but $P_{B b, g}(k)$ and $P_{B b, \ell}(k)$ cannot be zero individually for nonzero $u_{b}$. Note that, depending on the sign of $u_{b i}$, the balancing controller can virtually generate both positive (generate heat) and negative losses (consume heat) in each $\mathrm{Cell}_{i}$ to achieve balancing.

\section{Alternative Interpretation: Battery Emulation}

An alternative interpretation of the balancing controller is that it forms a time-varying virtual cell (balancing cell) in series with actual cell (voltage generating cell). The timevarying electrical parameters (virtual OCV, virtual capacity, and virtual resistance) of each virtual cell are given by

$$
v_{o c b, i}=v_{o c i} u_{b i}, \quad C_{e b, i}=\frac{C_{e i}}{u_{b i}}, \quad R_{e b, i}=R_{e i} u_{b i} .
$$

This can be easily verified by plugging in $u=u_{v}+u_{b}$ into the electro-thermal dynamics (4) and (8) of a cell. Since $v_{o c i}, C_{e i}$, and $R_{e i}$ are always positive and $u_{b i}$ can attain both positive and negative values, the virtual cell parameters can also attain both positive and negative values. In other words, the balancing controller forms a virtual positive/negative cell, which tries to compensate the actual cell by canceling out the imbalance in capacity, resistance and voltage of the actual cell. The electrical parameters of a compensated cell are given by

$$
\begin{gathered}
v_{o c i}^{c}=v_{o c i}\left(u_{v i}+u_{b i}\right), \quad C_{e i}^{c}=\frac{C_{e i}}{\left(u_{v i}+u_{b i}\right)}, \\
R_{e i}^{c}=R_{e i}\left(u_{v i}+u_{b i}\right) .
\end{gathered}
$$

\section{Simulation Setup}

\section{A. Some New Variables for Illustration Purpose}

To illustrate the balancing performance in next section, we use $\left\|e_{\xi}(k)\right\|_{\infty}$ and $\left\|e_{T_{s}}(k)\right\|_{\infty}\left(e_{\xi}\right.$ and $e_{T_{s}}$ are defined in (15) and (16)), which give the maximum SOC and temperature 
deviations (balancing errors) in the battery at any time instant. Similarly, to analyze the control behavior, we introduce average and rms cell currents over whole drive cycle given by

$$
\begin{aligned}
& I_{B a i}=f_{a}\left(i_{L}, u_{i}\right)=I_{B v a, i}+I_{B b a, i}, \\
& I_{B r i}^{2}=f_{r}\left(i_{L}, u_{i}\right)=I_{B v r, i}^{2}+I_{B b r, i}^{2}
\end{aligned}
$$

where functions

$$
\begin{aligned}
& f_{a}\left(i_{L}, u_{i}\right)=\frac{1}{N_{d}} \sum_{k=1}^{N_{d}} h \cdot i_{L}(k) u_{i}(k), \\
& f_{r}\left(i_{L}, u_{i}\right)=\frac{1}{N_{d}} \sum_{k=1}^{N_{d}} h \cdot i_{L}^{2}(k) u_{i}(k) .
\end{aligned}
$$

Here $I_{B v a, i}=f_{a}\left(i_{L}, u_{v i}\right)$ and $I_{B v r, i}^{2}=f_{r}\left(i_{L}, u_{v i}\right)$ are average and rms currents under $u_{v i}$ whereas $I_{B b a, i}=f_{a}\left(i_{L}, u_{b i}\right)$ and $I_{B b r, i}^{2}=f_{r}\left(i_{L}, u_{b i}\right)$ are virtual average and rms currents under $u_{b i}$ (note that $I_{B b r, i}$ is not a truly rms quantity as it can attain negative value for $u_{b i}<0$ ). These variables are used to study the functional properties of the complete controller, $u$, as well as its each component $\left(u_{v}\right.$ and $\left.u_{b}\right)$.

\section{B. Cell Data and String Configuration}

The modular battery considered for this simulation study consists of 4 modules, each containing one cell $(3.3 \mathrm{~V}, 2.3 \mathrm{Ah}$, A123 ANR26650M1A). The nominal values of cell's electrothermal parameters, shown in Table II, have been taken from [27]. The true cells are assumed to have capacity, resistance, and initial SOC variations (i.e. $C_{e i} \neq C_{e j}, R_{e i} \neq$ $R_{e j}$, and $\left.\xi_{i}(0) \neq \xi_{j}(0), \forall i, j\right)$ to thoroughly evaluate the controller performance. All other electro-thermal parameters are assumed equal. It is also realistic to assume that all cells have same initial temperature. There are various possible initial conditions of the battery depending on capacity, resistance, and initial SOC combination for each cell. For instance, for any two cells $\left(\mathrm{Cell}_{i}\right.$ and $\left.\mathrm{Cell}_{j}\right)$ in the modular battery, there are four possible cases:

- Case-1: $C_{e d, i}(0)<C_{e d, j}(0)$ and $R_{e i}<R_{e j}$,

- Case-2: $C_{e d, i}(0)>C_{e d, j}(0)$ and $R_{e i}<R_{e j}$,

- Case-3: $C_{e d, i}(0)<C_{e d, j}(0)$ and $R_{e i}>R_{e j}$,

- Case-4: $C_{e d, i}(0)>C_{e d, j}(0)$ and $R_{e i}>R_{e j}$.

Note that, during discharging, case- 1 and case- 4 are more challenging because a cell with higher resistance is also the one with higher initial dischargeable capacity. This implies conflicting cell usage requirements for thermal and SOC balancing. Therefore, it is interesting to consider a parametric variation profile of the modular battery, as shown in Fig. 4, where at least one cell pair in the string satisfies either case-1 or case-4. The true values of resistance, capacity, and initial SOC of cells are given below.

$$
\begin{aligned}
R_{e} & =\left[\begin{array}{llll}
11.7 & 13.3 & 16.1 & 16.8
\end{array}\right]^{\mathrm{T}} m \Omega, \\
C_{e} & =\left[\begin{array}{llll}
2.29 & 2.26 & 2.11 & 1.98
\end{array}\right]^{\mathrm{T}} A h, \\
\xi(0) & =\left[\begin{array}{llll}
0.75 & 0.78 & 0.88 & 0.90
\end{array}\right]^{\mathrm{T}} .
\end{aligned}
$$

The discrete time system matrices for this setup are obtained using Euler approximation with sampling interval $h=1$ second.
TABLE II

Cell Parameters And Controller Setting

\begin{tabular}{l|l|l|l}
\hline Parameter & Symbol & Value & Unit \\
\hline \multicolumn{3}{c}{ Cell Parameters } \\
\hline No. of Cells & $n$ & 4 & - \\
Nominal OCV & $v_{o c i}^{\star}$ & 3.3 & $V$ \\
Nominal Resistance & $R_{e i}^{\star}$ & 11.4 & $m \Omega$ \\
Nominal Capacity & $C_{e i}^{\star}$ & 2.3 & $A h$ \\
Thermal Resistance & $R_{u i}$ & $3.03, \forall i$ & $K W^{-1}$ \\
Heat Capacity & $C_{s i}$ & $71.50, \forall i$ & $J K^{-1}$ \\
Air Flow Rate & $\dot{V}_{f}$ & 0.0095 & $m^{3} s^{-1}$ \\
Air Thermal Conduct. & $c_{f}$ & 11.1105 & $W K^{-1}$ \\
Load Voltage Demand & $v_{L d}$ & 9.25 & $V$ \\
Inlet Fluid Temperature & $T_{f 0}$ & 25 & ${ }^{\circ} \mathrm{C}$ \\
OCV Vector & $v_{o c}$ & $v_{o c i}^{\star} 1_{n}$ & $V$ \\
\hline \multicolumn{3}{c}{ Controller Setting } \\
\hline SOC Deviation Allowance & $\delta \xi$ & $2.5 \%$ & - \\
Temp. Deviation Allowance & $\delta T_{s}$ & 1 & ${ }^{\circ} \mathrm{C}$ \\
Sampling Interval & $h$ & 1 & $\mathrm{~s}$ \\
Prediction Horizon & $N$ & 1 to 60 & $\mathrm{~s}$ \\
\hline
\end{tabular}

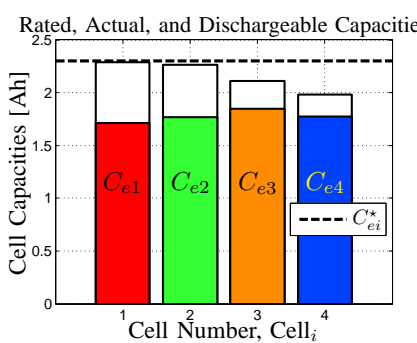

(a) Capacity distribution.

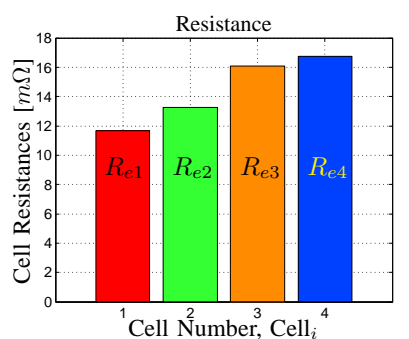

(b) Resistance distribution.
Fig. 4. Capacity and resistance distribution of cells. In Fig. 4(a), the level inside each container shows initial dischargeable capacity of cell.

\section{Drive Cycle Data and Battery Load Profile}

The balancing performance has been thoroughly evaluated under various driving behaviors/situations. Here, we show results particularly for three drive cycles namely

- $S C M 17 \mathrm{kmA6}$ [32]: It is a representative of low speed urban stop-n-go real world driving behavior (benign to mild) on a $17 \mathrm{~km}$ route in west Sweden.

- Standard ARTEMIS Rural: It is a representative of high speed rural driving behavior (normal to intensive).

- US06: It is a representative of very high speed highway driving behavior (aggressive).

The real battery current measurement data for SCM17kmA6 were obtained from Swedish Car Movement database [33], whereas the battery current data for ARTEMIS Rural and US06 were obtained by simulation of Toyota Prius PHEV in full EV mode in Advisor [34]. The scaled battery load current data and its histogram for each drive cycle are shown in Fig. 5. The demanded battery load voltage $v_{L d}(k)$, in this study, is assumed as a constant dc-link voltage of a three-phase twolevel inverter of Toyota Prius PHEV. It is chosen as 9.25 volts to satisfy condition (37) for all three drive cycles for the case of four cell battery considered here. 

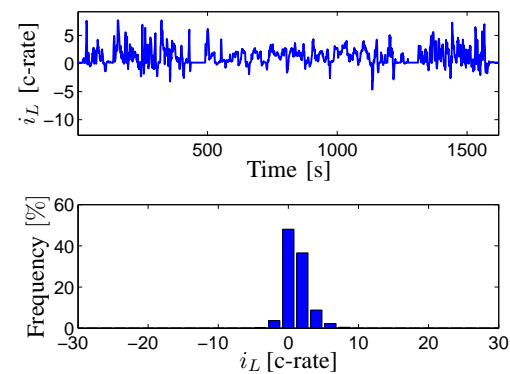

(a) SCM17kmA6: Measured battery current.
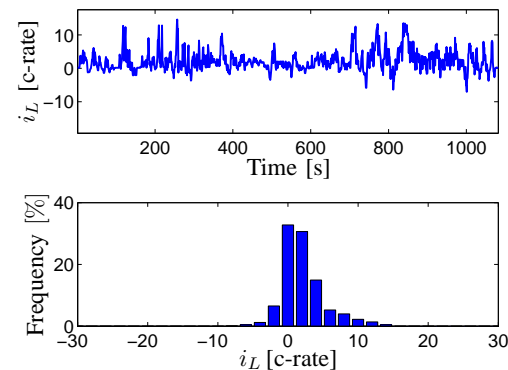

(b) ARTEMIS Rural: Simulated battery current.
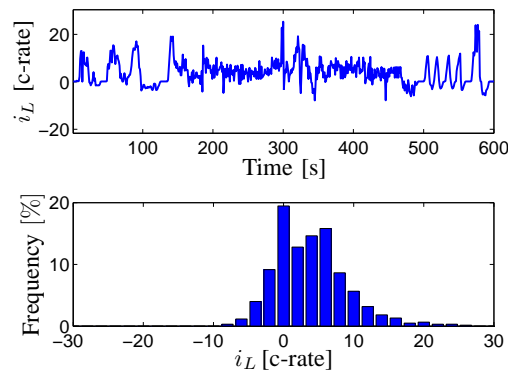

(c) USO6: Simulated battery current.

Fig. 5. Battery demanded load current (upper subplots) for three drive cycles and their histograms (lower subplots).

\section{Controller Setting and Tuning}

The balancing control objective is to bring maximum SOC deviation within $2.5 \%$ and keep temperature deviation within $1{ }^{\circ} \mathrm{C}$ all the time for all drive cycles. The main control parameters (penalty weights) have been tuned first using Bryson's rule [35, pg.537] and then trade-off weights $\gamma_{i}:$ s have been tweaked using iterative trial and error method to achieve the control objective with short prediction horizon $N$. For all tested drive cycles, $N=1$ gives satisfactory control performance. The longer horizons $(N=5-60)$ improves SOC balancing performance especially during aggressive driving.

\section{E. Solution Method}

The simulation study is based on the analytical solution (36) of voltage control problem and the numerical solution of balancing control problem (P-IV). To solve problem (P-IV), we used SeDuMi solver in CVX [28].

\section{Simulation Results and Discussion}

\section{A. One-step MPC Performance (No Model Mismatch)}

The balancing performance of the aged modular battery using ideal 1-step MPC (no parametric uncertainty) has been thoroughly investigated. The simulation results are shown in Fig. 6 for all three drive cycles. These plots are arranged in a $4 \times 3$ matrix of subfigures where each column corresponds to one of three drive cycles (SCM17kmA6, ARTEMIS Rural, and US06) and each row corresponds to one of four battery performance variables: $v_{L}(k), \xi(k), T_{s}(k)$, and $\left\{\left\|e_{\xi}(k)\right\|_{\infty},\left\|e_{T_{s}}(k)\right\|_{\infty}\right\}$. These plots clearly show that with passage of time, SOC deviation among cells is significantly reduced relative to the initial level of deviations. Similarly, temperature deviation stays within specified limits, despite significant deviation among cell resistances, during whole driving. Moreover, the cell balancing performance is achieved while simultaneously satisfying voltage constraint as shown in the first row. The controller particularly exhibits good thermal and SOC balancing performance for both SCM17kmA6 and ARTEMIS driving cycles as shown in first two columns.

The performance under US06 is not as good as for other driving cycles. The SOC deviation, as shown in Fig. 6(1), is nondecreasing during various intervals. It is primarily due to short duration, frequent aggressive (resulting in high $c$-rate) acceleration and braking, and long phases of nearly constant high speed driving during this cycle. For intensive loads (very high $c$-rates), the controller prioritizes thermal balancing to avoid fast increase in temperature of high resistance cells. Therefore, the controller particularly struggles with SOC balancing during each high $c$-rate acceleration pulse and also during constant high speed driving phases. However, the overall performance is still satisfactory as the maximum SOC balancing error has reduced from initial level of $7.8 \%$ to the level of $2.5 \%$ at final time and the maximum temperature deviation has remained within $1^{\circ} \mathrm{C}$ over whole driving cycle.

\section{B. Controller Behavior and Analysis}

To understand how the controller achieves the balancing, the various functional aspects of balancing controller are discussed in detail in the following subsections.

1) Balancing Controller: Virtual Charge and Heat Shuffler: Fig. 7 shows bar plots of average and rms currents, defined in (49a) and (49b), of each cell computed separately under voltage control, balancing control, and total control trajectories over whole drive cycle (note that the currents under $u_{v i}$ may exist in reality if balancing function is tuned off, but currents under $u_{b i}$ are only virtual). The figure is a $2 \times 3$ matrix of subfigures where the first row corresponds to average current and the second row corresponds to rms currents. Three columns corresponds to currents under $u_{v}, u_{b}$, and $u$ respectively. The results are presented here for ARTEMIS only as one case-study is sufficient for current purpose.

The voltage controller decides almost equal average current (or power) for each cell as shown in Fig. 7(a). This is of course not optimal as cells have different dischargeable capacities as shown in Fig. 4(a). Therefore, the balancing controller performs corrective action in average currents as shown in Fig. 7(b). In a nutshell, the controller achieves SOC balancing by (virtually) taking out electric charge from cells with higher dischargeable capacities $\left(\mathrm{Cell}_{3}\right.$ and $\left.\mathrm{Cell}_{4}\right)$ and delivering it to cells with lower dischargeable capacities $\left(\mathrm{Cell}_{1}\right.$ and $\mathrm{Cell}_{2}$ ). Note that after shuffling of charges, the average current distribution now resembles the dischargeable capacity distribution (compare Fig. 7(c) with 4(a)).

Similarly, the voltage controller decides almost equal level of rms current for all cells as shown in Fig. 7(d). This is again not optimal as cells have different resistances as shown 

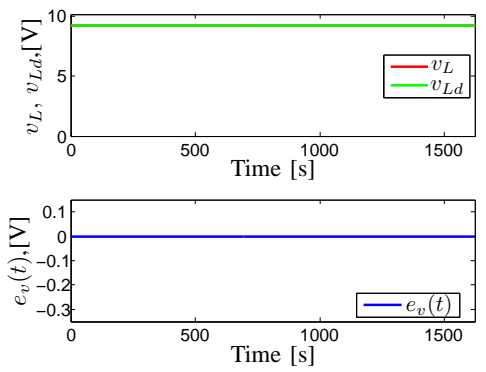

(a) Voltage response and error.

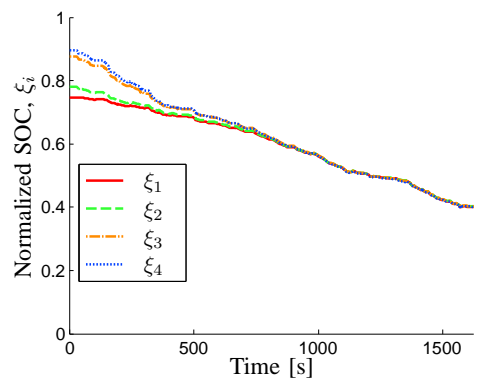

(d) SOC balancing performance.

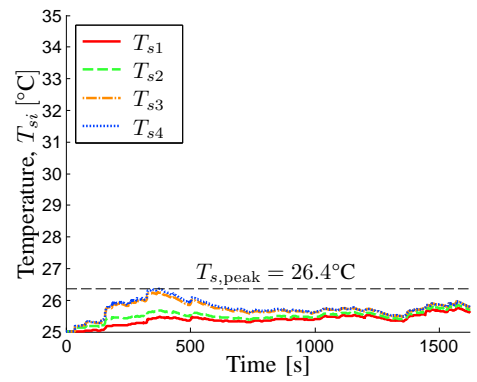

(g) Thermal balancing performance.
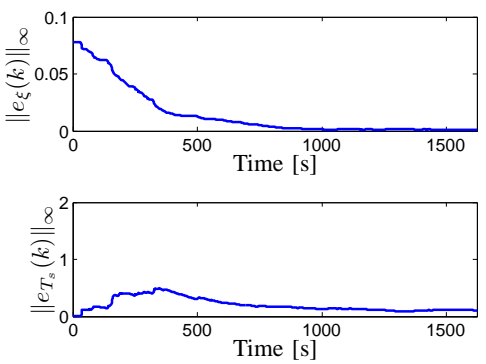

(j) Evolution of balancing errors
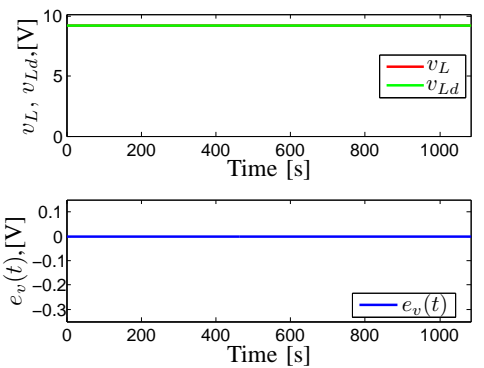

(b) Voltage response and error.

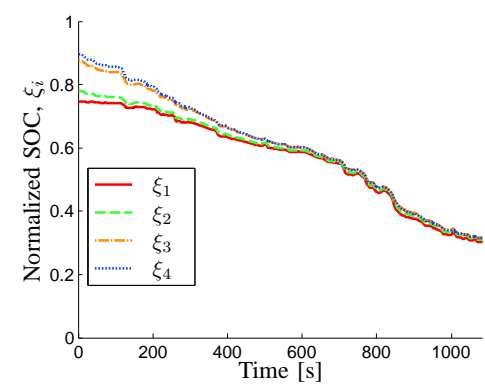

(e) SOC balancing performance.

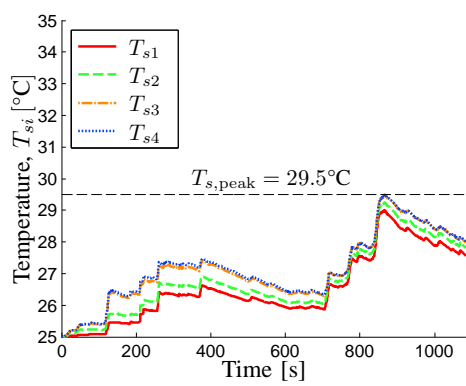

(h) Thermal balancing performance.
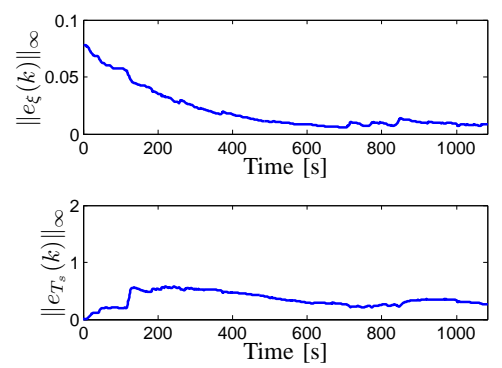

(k) Evolution of balancing errors.
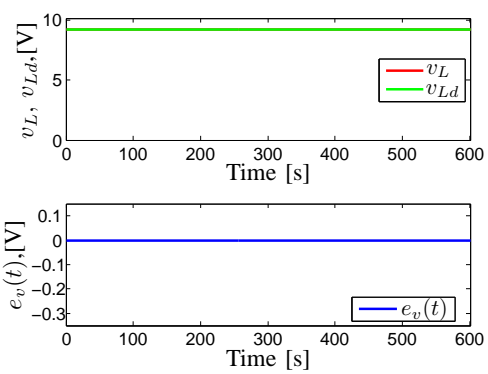

(c) Voltage response and error.

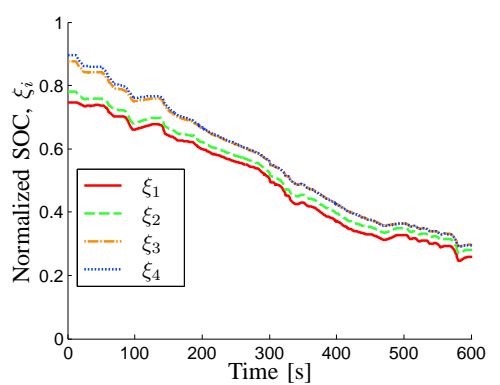

(f) SOC balancing performance.

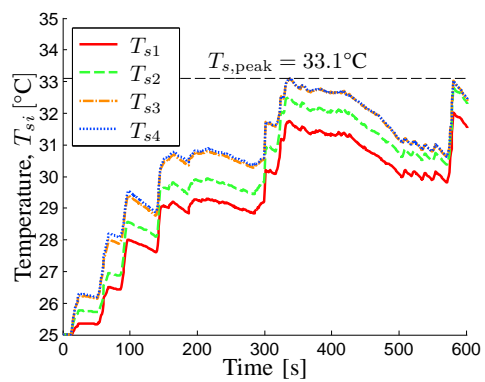

(i) Thermal balancing performance.
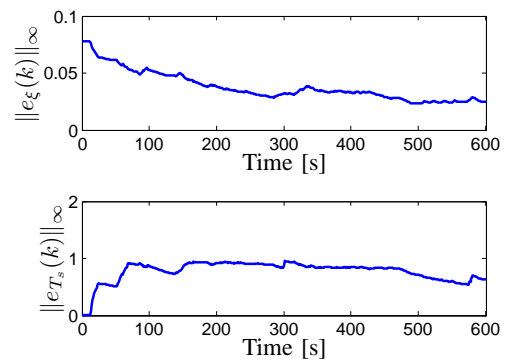

(1) Evolution of balancing errors.

Fig. 6. Simulation results for 1-step MPC performance under three drive cycles is shown: SCM17kmA6: first column; ARTEMIS Rural: second column; and US06: third column. These figures show that the driving behavior may have significant impact on the cell balancing performance.

in Fig. 4(b). The balancing controller in this case performs corrective action in rms current as shown in Fig. 7(e). In a nutshell, the controller achieves thermal balancing by (virtually) taking out heat from cells with higher resistances $\left(\mathrm{Cell}_{3}\right.$ and $\mathrm{Cell}_{4}$ ) and delivering it to cells with lower resistances $\left(\mathrm{Cell}_{1}\right.$ and $\left.\mathrm{Cell}_{2}\right)$. Note that after shuffling of losses, the rms current distribution resembles the mirror image of resistance distribution (compare Fig. 7(f) with 4(b)).

In the light of above discussion, it is now quite obvious that the balancing controller emulates a charge and heat shuffler.
It slightly shuffles cell charges and power losses to achieve thermal and SOC balancing objectives simultaneously. It is also noteworthy that balancing is achieved with very small corrections indeed $(< \pm 10 \%$ of maximum average and rms currents, see figures 7(b) and 7(e)), where the corrections in rms currents are opposite to those in average currents.

2) Balancing Controller: Virtual Power Redistributor: To understand the controller's instantaneous behavior, we study the histograms of the instantaneous terminal powers of each cell as shown in Fig. 8. The load can be classified into various 


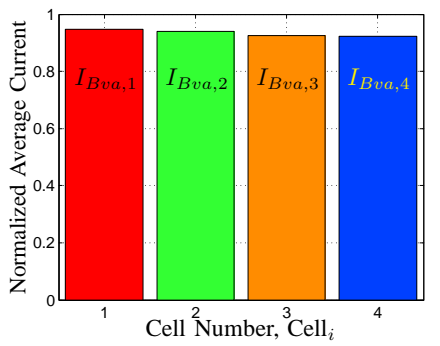

(a) Cell average currents under $u_{v}$.

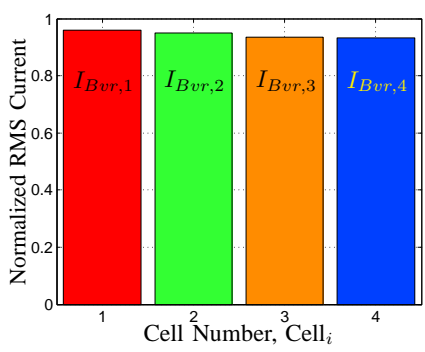

(d) Cell rms currents under $u_{v}$.

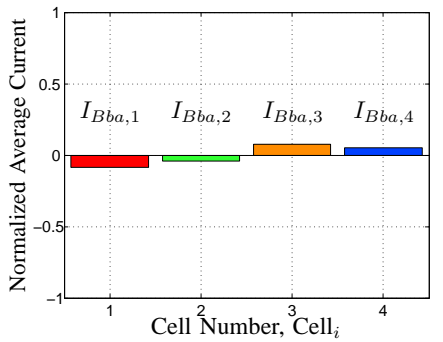

(b) Cell average currents under $u_{b}$.

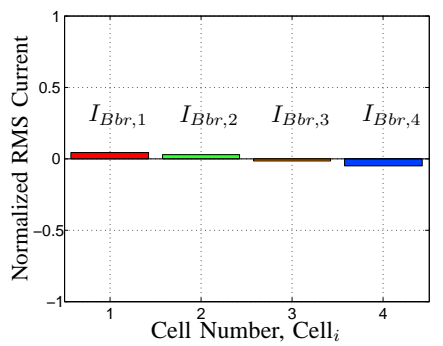

(e) Cell rms currents under $u_{b}$.

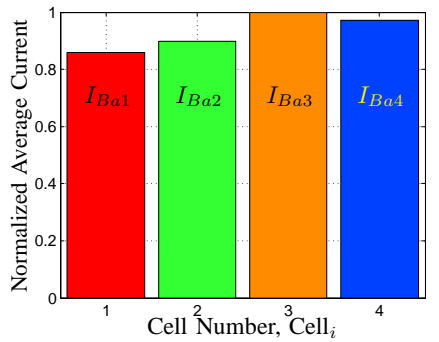

(c) Cell average currents under $u$.

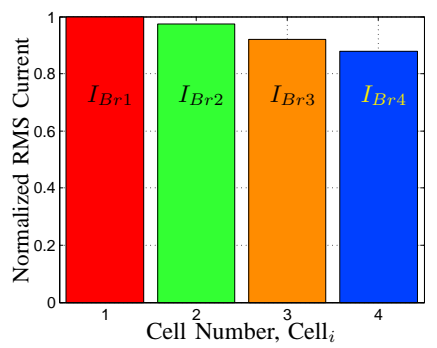

(f) Cell rms currents under $u$.

Fig. 7. Cell average and rms current behavior for ARTEMIS drive cycle under $u_{v}, u_{b}$, and $u$. Note corrective action taken by balancing controller $u_{b}$. The balancing controller slightly shuffles $(< \pm 10 \%)$ cell charges and heat to achieve thermal and SOC balancing simultaneously.

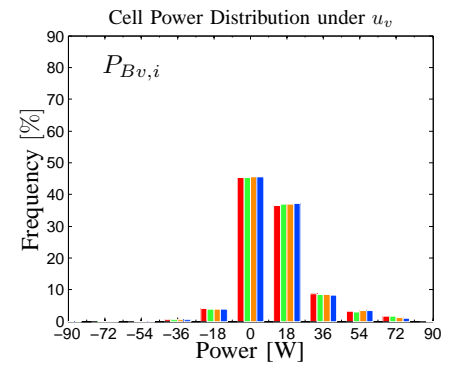

(a) Each cell power under $u_{v}$.

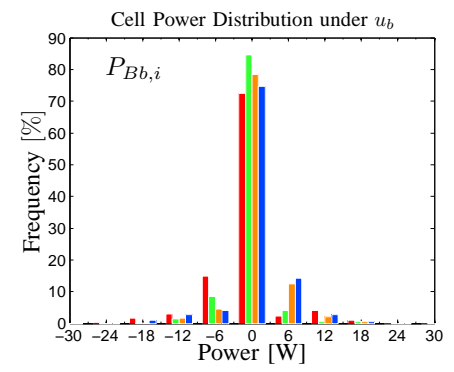

(b) Each cell power under $u_{b}$.

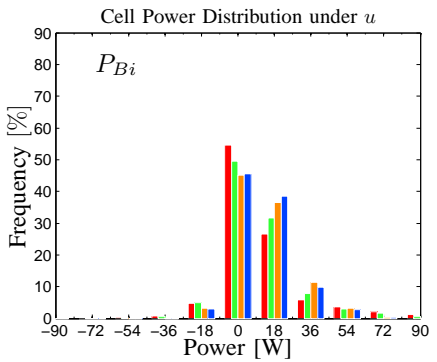

(c) Each cell power under $u$.

Fig. 8. Histograms of instantaneous powers of cells for ARTEMIS drive cycle under $u_{v}, u_{b}$, and $u$, where each group of four bars correspond to four cells. Note that the balancing controller acts as virtual power redistributor to reshape the histogram of each cell in each power bin.

types depending on its frequency of occurrence and power level. Therefore, we can roughly say that each power bin in these histograms corresponds to a certain type of load. The key to achieve simultaneous thermal and SOC balancing is to optimally decide the duty of each cell in each power bin. The voltage controller unjustly decides almost identical power distribution among cells in each power bin as shown in Fig. 8(a). The balancing controller acts as a virtual power redistributor to reshape the histogram of each cell as shown in Fig. 8(b). It performs corrective actions by slightly redistributing the load on each cell according to its resistance and dischargeable capacity. For example, the balancing controller takes following corrective actions for various types of loads:

- It shifts infrequent high-power driving loads (54,72, and 90 watt power bins) to cells 1 and 2, which have lower resistances. This type of short duration high power loads result in higher rms but small average currents. Therefore, this control action saves cells 3 and 4, which have higher resistances, from faster heating and temperature deviation while securing cells 1 and 2 from faster discharge.
- It shifts more frequent intermediate-power driving load (18 and 36 watt bins) to cells 3 and 4, which have higher dischargeable capacities. This type of relatively long duration intermediate loads result in higher average current without significant increase in heating value of current. This particular control action saves cells 1 and 2 from faster discharging and SOC deviation while keeping temperature deviation of cells 3 and 4 within limits.

- It shifts highly frequent low-power mixed load (power range around zero watt) to cells 1 and 2 because this type of load contains a lot of regenerative energy, which helps cells 1 and 2 to correct their charge levels.

- It distributes the infrequent mild-power regenerations among all cells as per their dischargeable capacities.

- It uses cells 1 and 2 during highly infrequent high-power regenerations. This action saves cells 3 and 4 from extra heating and also helps in balancing SOC among cells.

This set of control actions in different power bins results in different load sharing patterns for different classes of load. These nonuniform load sharing patterns enable the simulta- 
neous thermal and SOC balancing. We can roughly say that cells 1 and 2 are used more during high-power loads and regeneration phases, whereas cells 3 and 4 are used more in low to medium driving power ranges.

Remark 2. In the light of the above discussion, it is easy to conclude that the load variations play a big role in achieving simultaneous thermal and SOC balancing under unipolar battery control mode. The difference in average and rms current distributions (compare figures $7(c)$ and $7(f))$ is only possible due to variations in the load profile. The load variations (i.e. blend of low and high c-rates) allow somewhat independent adjustment of average and rms currents, which is a key for simultaneous thermal and SOC balancing. If the load current is continuously high (only one load type or power bin) then the simultaneous balancing is not possible because the controller can only perform one type of corrective actions in such a power bin i.e. it prioritizes thermal balancing without caring much about SOC balancing.

\section{Control Performance: Long Versus Short Horizons}

Now we compare the performance of 60-step and 1-step MPCs particularly for US06 as it is relatively hard to handle using 1-step MPC. The results are shown in Fig. 9.

The SOC balancing speed has increased as shown in upper subplot of Fig. 9. Due to faster response, there is now $1 \%$ final SOC imbalance ( $1.5 \%$ less compared to that with 1-step MPC). This improvement appears because the controller can now do better planning due to large accessible information about future energy flows into/out of battery pack. Depending on the direction of energy flows, cells can be both charged (increase SOC) and discharged (decrease SOC). If we know at current time that there will be a certain level of regenerative energy in near future then we can afford to discharge a certain cell more momentarily and charge it again during regeneration. Therefore, instead of instantaneous short-sighted actions, it may be beneficial to look ahead in future for upcoming regeneration phases to provide relatively better cell duty schedule for SOC balancing. This long-term planning is particularly useful for aggressive driving cycles like US06.

The thermal balancing under 60-step MPC, shown in lower subplot of Fig. 9, has improved only slightly in terms of mean temperature deviation $\left(\frac{1}{N_{d}} \sum_{k=1}^{N_{d}}\left\|e_{T_{s}}(k)\right\|_{\infty}\right)$ over full drive cycle $\left(0.71^{\circ} \mathrm{C}\right.$ versus $0.77^{\circ} \mathrm{C}$ under 1 -step MPC). The first reason is that temperature rises quickly only during intensive load demand (aggressive acceleration/braking). During these short high power pulses, the controller must take corrective action instantaneously to keep temperature deviation small. The second reason is that the energy recuperation is not beneficial for thermal balancing because cells always dissipate (never consume) heat regardless of current direction. Therefore, the long-term planning is not that crucial directly for thermal balancing. However, note that the faster decay of initial SOC imbalance under 60-step MPC may enable the controller to prioritize thermal balancing sooner than that under 1-step MPC. Therefore, the longer prediction horizon may also indirectly benefit thermal balancing, see second half of the driving in Fig. 9.
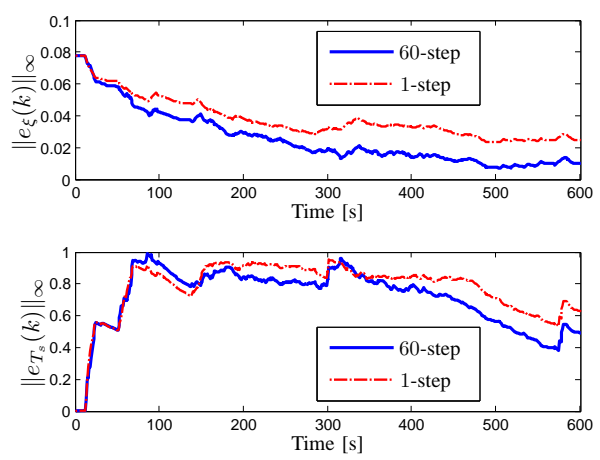

Fig. 9. Longer Prediction Horizon: Balancing performance comparison between 60-step MPC and 1-step MPC for US06 drive cycle.
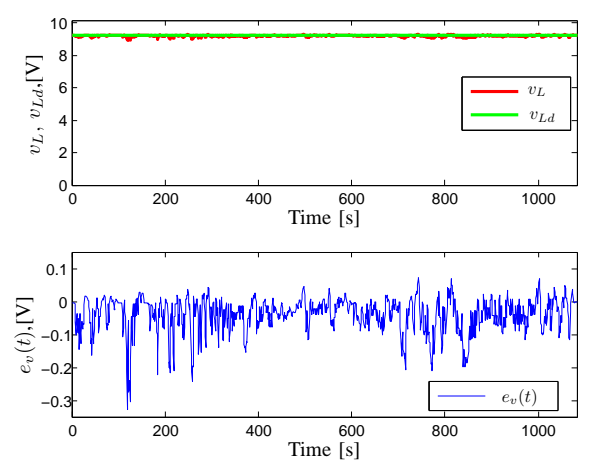

(a) Voltage response and error.
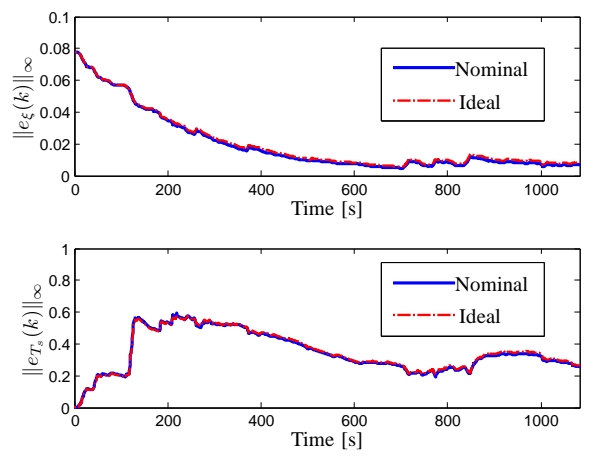

(b) Evolution of balancing errors.

Fig. 10. Robust control performance under parametric uncertainty and resistance variation with temperature.

\section{Control Robustness under Model Mismatch}

Now let us consider an uncertain battery model to investigate the control robustness. For this purpose, a nominal 1-step MPC (designed assuming nominal cell model with constant parameters, but perfectly known battery state) is simulated with the true battery that has parametric imbalance among its cells (up to $46 \%$ in cell resistance and $15 \%$ in capacity) as shown in Fig. 4. In addition, each cell resistance also varies with temperature as shown in Fig. 3. The simulation results for ARTEMIS drive cycle are shown in Fig. 10. The terminal voltage error as shown in Fig. 10(a) is very small $(<3.2 \%)$ as predicted by sensitivity relation (44). This error can be further reduced using gain-scheduling, which would then require es- 
timation of cell resistance. The balancing performance, shown in Fig. 10(b), has no noticeable difference from the ideal case shown in Fig. 6(k) (plotted here again in red) despite the large parametric uncertainty. This suggests that the controller is robust to parametric uncertainty and small resistance variation in normal operating temperature range $[25,40]^{\circ} \mathrm{C}$, which is normally maintained by active cooling of batteries in $\mathrm{xEVs}$. To exactly characterize the robustness property, in terms of range of parametric uncertainty and resistance variation with temperature, requires further investigation, which is beyond the scope of this study.

\section{Summary and CONClusions}

The main purpose of this paper was to devise a predictive control scheme for terminal voltage control and simultaneous thermal and SOC balancing of batteries using minimum future load information. This problem boils down to load management i.e. deciding the power flow into/out of each cell according to its state. For this, we have proposed an LQ MPC scheme for the MLC-based modular battery with parametric imbalance among its cells. The control scheme has been developed using orthogonal decomposition of controller into two components, one for voltage control and the other for balancing control. The voltage controller strictly satisfies the voltage demand, but distributes the power almost equally among all modules. The balancing controller achieves balancing by correcting the power distribution without disturbing the voltage. The balancing performance has been thoroughly evaluated for various prediction horizons. In particular, the 1-step MPC has been carefully tested to assess the balancing potential for most realistic cases where no future load information is accessible. The results have been presented for three drive cycles (realworld SCM17kmA6, ARTEMIS Rural, and US06) to analyze the balancing performance under various driving situations. In short, the performance highly depends on the level of load variations. Any drive cycle with long high-power intervals is challenging for simultaneous thermal and SOC balancing.

The results show that it is sufficient to use 1-step MPC to achieve promising thermal and SOC balancing performance for benign to normal driving with short driving pulses (as in SCM17kmA6 and ARTEMIS Rural drive cycles). The 1-step MPC also shows good thermal balancing performance under aggressive highway driving (i.e. long driving pulses with high acceleration and speed as in US06). The SOC balancing is not as good as that under benign/normal driving, but is still acceptable. It can be further improved, if needed, by using longer prediction horizon. We have also analyzed the sensitivity and robustness of nominal controller to modeling uncertainty and variation of cell resistances with temperature. These variations have no noticeable effect on balancing performance and also generate negligible $(<3.2 \%)$ voltage errors.

The control performance shown by 1-step MPC is quite encouraging for real-time control implementation. However, the experimental validation is still required for large battery packs. In addition, an appropriate module size and the extra losses due to additional electronic components (power switches and gate drivers for each module) require thorough investigation to assess the overall benefit of the modular battery. Nevertheless, the proposed control scheme has given us deeper insight into structural and functional properties of the simultaneous thermal and SOC balancer, which can be useful for its simple rule-based implementation in our future work.

\section{APPENDIX}

The matrices for model (11a)-(11b) are given by

$$
\begin{aligned}
& A=\left[\begin{array}{cc}
A_{E} & 0 \\
0 & A_{\vartheta}
\end{array}\right], B\left(i_{L}(t)\right)=\left[\begin{array}{c}
B_{E} i_{L} \\
B_{\vartheta} i_{L}^{2}
\end{array}\right], \\
& A_{E}=0_{n \times n}, B_{E}=-\operatorname{diag}\left(b_{e 1}, \cdots, b_{e n}\right) \in \mathbb{R}^{n \times n}, \\
& A_{\vartheta}=\left[\begin{array}{cc}
A_{T} & W_{T} \\
0_{n}^{\mathrm{T}} & 0
\end{array}\right], \quad B_{\vartheta}=\left[\begin{array}{c}
B_{T} \\
0_{n}^{\mathrm{T}}
\end{array}\right] \text {, } \\
& A_{T}=\left[a_{t i j}\right] \in \mathbb{R}^{n \times n}, \quad B_{T}=\operatorname{diag}\left(b_{t 1}, \cdots, b_{t n}\right) \in \mathbb{R}^{n \times n}, \\
& W_{T}=\left[\begin{array}{lll}
w_{t 1} & \cdots & w_{t n}
\end{array}\right]^{\mathrm{T}} \in \mathbb{R}^{n} \text {, } \\
& C=\left[\begin{array}{cc}
0 & I_{n+1} \\
0_{n}^{\mathrm{T}} & 0_{n+1}^{\mathrm{T}}
\end{array}\right], D\left(i_{L}(t)\right)=\left[\begin{array}{c}
0 \\
D_{v}(t)
\end{array}\right], \\
& D_{v}(t)=\left[\begin{array}{lll}
d_{v 1}(t) & \cdots & d_{v n}(t)
\end{array}\right] \in \mathbb{R}^{1 \times n},
\end{aligned}
$$

where $A_{T}$ is a constant lower triangular thermal subsystem matrix and the coefficients $a_{t i j}$ and $w_{t i}$ are thermal circuit parameters given by (see [17] for derivation of these relations)

$$
\begin{aligned}
a_{t i j}= \begin{cases}\left(\frac{\prod_{k=(j+1)}^{(i-1)} \beta_{k}}{\prod_{k=j}^{(i-1)} \alpha_{k}}\right) a_{s i}, & \forall i>j, \\
-a_{s i}, & \forall i=j \geq 1, \\
0, & \forall i<j,\end{cases} \\
w_{t i}=-\sum_{j=1}^{i} a_{t i j} \quad \forall i \geq 1,
\end{aligned}
$$

for coolant flow from $\mathrm{Cell}_{1}$ towards $\mathrm{Cell}_{i}$, where $\alpha_{i}, \beta_{i}, a_{s i}$, and various other parameters are defined in Table I. The coefficients $b_{e i}=\frac{1}{3600 C_{e i}}$ and $b_{t i}=\frac{R_{e i}}{C_{s i}}$. Note that $D_{v}(t)$ is a feedthrough gain from $u(t)$ to $v_{L}(t)$.

\section{REFERENCES}

[1] S. J. Moura, "Techniques for battery health conscious power management via electrochemical modeling and optimal control," Ph.D. dissertation, University of Michigan, 2011.

[2] C. D. Rahn and C.-Y. Wang, Battery systems engineering. John Wiley \& Sons, 2012.

[3] A. Barré, B. Deguilhem, S. Grolleau, M. Gérard, F. Suard, and D. Riu, "A review on lithium-ion battery ageing mechanisms and estimations for automotive applications," Journal of Power Sources, vol. 241, no. 0, pp. $680-689,2013$.

[4] J. Vetter, P. Novak, M. Wagner, and et.el., "Ageing mechanisms in lithium-ion batteries," Journal of power sources, vol. 147, no. 1, pp. 269-281, 2005.

[5] J. Wang, P. Liu, Hicks-Garner, and et.el., "Cycle-life model for graphite$\mathrm{LiFePO}_{4}$ cells," Journal of Power Sources, vol. 196, no. 8, pp. 39423948, 2011.

[6] J. Groot, State-of-Health Estimation of Li-ion Batteries: Ageing Models, ser. PhD Thesis. New Series, no: 3815. Chalmers University of Technology, 2014.

[7] L. Lu, X. Han, J. Li, J. Hua, and M. Ouyang, "A review on the key issues for lithium-ion battery management in electric vehicles," Journal of Power Sources, vol. 226, pp. 272-288, 2013.

[8] F. Altaf, L. Johannesson, and B. Egardt, "Simultaneous Thermal and State-of-Charge Balancing of Batteries: A Review," in Vehicle Power and Propulsion Conference (VPPC), 2014 IEEE, Oct. 2014, pp. 1-7. 
[9] T. M. Bandhauer, S. Garimella, and T. F. Fuller, "A critical review of thermal issues in lithium-ion batteries," Journal of the Electrochemical Society, vol. 158, no. 3, pp. R1-R25, 2011.

[10] B. Wu, V. Yufit, M. Marinescu, G. J. Offer, R. F. Martinez-Botas, and N. P. Brandon, "Coupled thermal-electrochemical modelling of uneven heat generation in lithium-ion battery packs," Journal of Power Sources, 2013.

[11] R. Mahamud and C. Park, "Reciprocating air flow for li-ion battery thermal management to improve temperature uniformity," Journal of Power Sources, vol. 196, no. 13, pp. 5685 - 5696, 2011.

[12] M. Dubarry, N. Vuillaume, and B. Y. Liaw, "Origins and accommodation of cell variations in li-ion battery pack modeling," International Journal of Energy Research, vol. 34, no. 2, pp. 216-231, 2010.

[13] V. Viswanathan and M. Kintner-Meyer, "Second use of transportation batteries: Maximizing the value of batteries for transportation and grid services," Vehicular Technology, IEEE Transactions on, vol. 60, no. 7, pp. 2963-2970, Sept 2011.

[14] J. Gallardo-Lozano, Romero-Cadaval, and et.el., "Battery equalization active methods," Journal of Power Sources, vol. 246, pp. 934-949, 2014

15] J. Cao, N. Schofield, and A. Emadi, "Battery balancing methods: A comprehensive review," in Vehicle Power and Propulsion Conference, 2008. VPPC '08. IEEE, sept. 2008, pp. $1-6$.

[16] W. Bentley, "Cell balancing considerations for lithium-ion battery systems," in Battery Conference on Applications and Advances, 1997., 12th Annual, jan 1997, pp. $223-226$.

[17] F. Altaf, L. Johannesson, and B. Egardt, "On Thermal and State-ofCharge Balancing using Cascaded Multi-level Converters," Journal of Power Electronics, vol. 13, no. 4, pp. 569-583, July 2013.

[18] F. Altaf, Thermal and State-of-Charge Balancing of Batteries using Multilevel Converters. Licentiate Thesis, Chalmers University of Technology, 2014. [Online]. Available: http://publications.lib.chalmers. se/records/fulltext/194660/194660.pdf

[19] F. Altaf, L. Johannesson, and B. Egardt, "Evaluating the Potential for Cell Balancing using a Cascaded Multi-Level Converter using Convex Optimization," in IFAC Workshop on Engine and Powertrain Control, Simulation and Modeling, 2012, Oct. 2012.

[20] J. Barreras, C. Pinto, and et.al., "Multi-objective control of balancing systems for li-ion battery packs: A paradigm shift?" in Vehicle Power and Propulsion Conference (VPPC), 2014 IEEE, Oct. 2014.

[21] M. Malinowski, K. Gopakumar, J. Rodriguez, and M. Pérez, "A survey on cascaded multilevel inverters," Industrial Electronics, IEEE Transactions on, vol. 57, no. 7, pp. $2197-2206$, july 2010.

[22] J. Rodriguez, L. Franquelo, S. Kouro, J. Leon, R. Portillo, M. Prats, and M. Perez, "Multilevel converters: An enabling technology for highpower applications," Proceedings of the IEEE, vol. 97, no. 11, pp. 1786 -1817 , nov. 2009.

[23] J. Rawlings, "Tutorial overview of model predictive control," Contro Systems, IEEE, vol. 20, no. 3, pp. 38-52, Jun 2000.

[24] X. Hu, S. Li, and H. Peng, "A comparative study of equivalent circuit models for li-ion batteries," Journal of Power Sources, vol. 198, no. 0, pp. $359-367,2012$

[25] X. Lin, H. E. Perez, S. Mohan, J. B. Siegel, A. G. Stefanopoulou, Y. Ding, and M. P. Castanier, "A lumped-parameter electro-therma model for cylindrical batteries," Journal of Power Sources, vol. 257, pp. $1-11,2014$.

[26] X. Lin, H. Fu, H. E. Perez, and et.el., "Parameterization and observability analysis of scalable battery clusters for onboard thermal management," Oil \& Gas Science and Technology-Revue d'IFP Energies nouvelles, vol. 68, no. 1, pp. 165-178, 2013.

[27] X. Lin, H. Perez, J. Siegel, A. Stefanopoulou, and et.el., "Online parameterization of lumped thermal dynamics in cylindrical lithium ion batteries for core temperature estimation and health monitoring," Contro Systems Technology, IEEE Transactions on, vol. 21, no. 5, pp. 1745 1755, Sept 2013.

[28] M. Grant and S. Boyd, "CVX: Matlab software for disciplined convex programming, version 1.21," Apr 2011.

[29] M. Grant, S. Boyd, and Y. Ye, "Disciplined convex programming," in Global Optimization: From Theory to Implementation, Nonconvex Optimization and Its Application Series. Springer, 2006, pp. 155-210.

[30] S. Boyd and L. Vandenberghe, Convex Optimization. Cambridge University Press, 2006

[31] A. J. Laub, Matrix analysis for scientists and engineers. Siam, 2005.
[32] S. Karlsson, "The swedish car movement data project final report." Tech. Rep., 2013, 81.

[33] Test Site Sweden, "Vehicle research database," 2014 [Online]. Available: http://www.testsitesweden.eu/environment/ vehicle-research-database

[34] K. Wipke, M. Cuddy, and S. Burch, "Advisor 2.1: a user-friendly advanced powertrain simulation using a combined backward/forward approach," Vehicular Technology, IEEE Transactions on, vol. 48, no. 6 , pp. 1751-1761, Nov 1999.

[35] G. F. Franklin, J. D. Powell, and A. Emami-Naeini, Feedback control of dynamics systems, 4th ed. Prentice Hall, NJ, 2002.

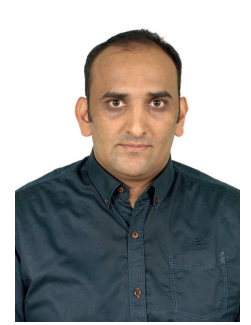

Faisal Altaf received his BE degree in Mechatronics from the National University of Sciences and Technology (NUST), Pakistan, in 2004 and his M.Sc. degree in Electrical Engineering from the $\mathrm{KTH}$ Royal Institute of Technology, Sweden, in 2011. From 2004 to 2008, he worked as an R\&D/Contro Engineer in Microwave Engineering Research Lab at NUST where he was heavily involved in the development of a radar system. From 2010 to 2011 , he was a Research Engineer in the Department of Automatic Control at KTH where he worked on control over wireless networks. He is currently pursuing the $\mathrm{Ph} . \mathrm{D}$. degree in Automatic Control at Chalmers University of Technology, Sweden. His research interests are at the intersection of constrained optimal control, convex optimization, batteries, and power electronics with special focus on mode predictive control and battery management systems in the automotive area.

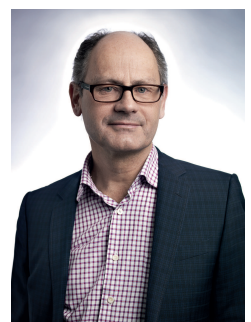

Bo Egardt received the M.Sc. degree in electrical engineering and the Ph.D. degree in automatic control from Lund Institute of Technology, Sweden, in 1974 and 1979, respectively. During 1980, he was a Research Associate at the Information Systems Laboratory, Stanford, CA. From 1981 to 1989 , he was with Asea Brown Boveri, where he was heavily involved in the introduction of adaptive control in the process industry. In 1989, he was appointed Professor of automatic control at Chalmers University of Technology, Sweden. His main areas of interest include adaptive and hybrid control and applications of control in the automotive area. Dr. Egardt has been an Associate Editor of IEEE Transactions on Control Systems Technology and of the European Journal of Control. He is a member of the editorial board for the International Journal of Adaptive Control and Signal Processing. He is a member of the Royal Swedish Academy of Engineering Sciences and a Fellow of the IEEE.

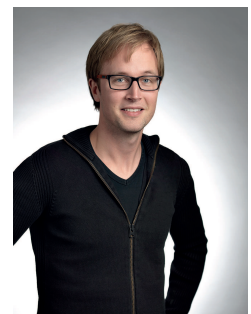

Lars Johannesson Mårdh received his M.Sc. degree in Automation and Mechatronics and his Ph.D. in Automatic Control from the Chalmers University of Technology in Gothenburg, Sweden in 2004 and in 2009, respectively. From 2009 to 2014 , he was a Researcher at Chalmers and the Viktoria Swedish ICT where he worked on powertrain control of hybrid and plug-in hybrid electric vehicles, control of auxiliary systems in trucks, battery management, and system studies of hybrid vehicles. Since 2014 , he is with the Volvo Car Corporation where he is developer of the lateral control system that is used in Volvo's self-driving car projects and driver support functions. His main research interests include optimal control, model predictive control, convex optimization, and their application in the automotive area. 